\title{
Tidal encounters of ellipsoidal granular asteroids with planets
}

\author{
Ishan Sharma* James T. Jenkins Joseph A. Burns ** \\ Department of Theoretical and Applied Mechanics \\ Cornell University, Ithaca, NY 14853
}

\begin{abstract}
We investigate planetary fly-bys of asteroids using an approximate volume-averaged method that offers a relatively simple, but very flexible, approach to study the rotational dynamics of ellipsoids. The asteroid is considered to be a deformable, prolate ellipsoid, with its interior being modelled as a rigid-granular material. Effects due to the asteroid's rotation, its self-gravity and gravitational interaction with the planet are included. Using a simplified approach allows us to explore in detail the mechanics of asteroid's deformations and disruptions during planetary encounters. We also compare our results with those obtained by Richardson et al. (1998) who used a large numerical code. We find that many of the features reported by them can indeed be captured by our rather simple methodology, and we discuss the reasons why some of our results differ from theirs.
\end{abstract}

\section{Introduction}

Evidence is accumulating that asteroids might be granular aggregates lacking tensile strength (Richardson et al. 1998, 2002) that are simply held together by self-gravity alone. Such objects may be so fragile that they fragment during planetary fly-bys owing to tidal effects (Sridhar and Tremaine 1992, Asphaug et al. 1994, Richardson et al. 1998, Walsh and Richardson 2006). In fact, such an event was observed when comet Shoemaker-Levy 9 flew past Jupiter. Presumably asteroids that are thought to have accumulated by gravitational processes like those that brought comets together, and which might be shattered over their lifetimes by energetic collisions (Holsapple et al. 2002), may

* Now at ITG, DAMTP, Cambridge University, UK.

**Also at the Department of Astronomy, Cornell University.

Email address: is42@cornell.edu (Ishan Sharma). 
behave similarly. A study into the nature of tidal break-up may provide clues to the asteroid's interior. For example, the observation of a disruption event, where one is predicted during a planetary flyby, will reinforce the belief that asteroids are granular aggregates. By contrast, no observed disruption, under circumstances when one is forecast, will presumably mean that the asteroid has some tensile strength. Such insights would be useful in understanding the size distribution and duplicity of Near-Earth Objects (NEOs) as well as the prevalence of binary craters on the terrestrial planets.

Tidal break-up during planetary passage has previously been approached by detailed particle simulations. We will investigate whether volume-averaged, (semi)-analytical methods may offer a much simpler way to analyze planetary fly-bys and may, simultaneously, provide a deeper insight into the relevant mechanics. To some degree, this was pursued earlier by Sridhar and Tremaine (1992), whose analysis, however, differs from ours in several respects. First, they considered a fluid-like material response with a pressure-dependent viscosity. This particular form of viscosity is quite artificial, as the authors themselves admit, but seems to give "reasonable" results. Secondly, they consider only parabolic fly-by paths; these have longer fly-by times than hyperbolic encounters. This, in turn, augments tidal interaction, thereby increasing the probability of break-ups. Finally, they do not investigate the effects of variables, such as the long-axis's orientation and tidal torques in the vicinity of the periapse, on the fly-by's possible outcomes.

In a pioneering numerical study, Richardson et al. (1998) considered the breakup of bodies as they flew past planets, with the fragmentation occurring due to the combined effects of rotation, tidal torques (in case of asymmetric bodies) and tidal stresses. They modeled the body as a granular aggregate comprised of 247 smooth spheres that interacted with each other only through inelastic collisions and that were held together just by gravity. A large numerical simulation was used to determine the motion of individual spheres. Various parameters, including the initial angular velocity vector and encounter variables, were changed to explore the consequences of different fly-bys.

In the analysis to follow, the above problem is solved using a volume-averaged method. We will demonstrate that much of the qualitative behavior obtained by Richardson et al. (1998) can be achieved far more easily by this technique, thereby allowing many different materials to be investigated. We also discuss several reasons for those discrepancies that are seen between our results and those of Richardson et al. (1998). Furthermore, the simplicity of our approach allows us to explore the mechanics of tidal encounters in detail. Because little is known of an asteroid's interior, more detailed modelling at this stage is, perhaps, unwarranted. In fact, the ideal way to gain insight into the essential mechanics is to keep the number of free parameters at a minimum while simultaneously still being able to produce the essential aspects of the observed 


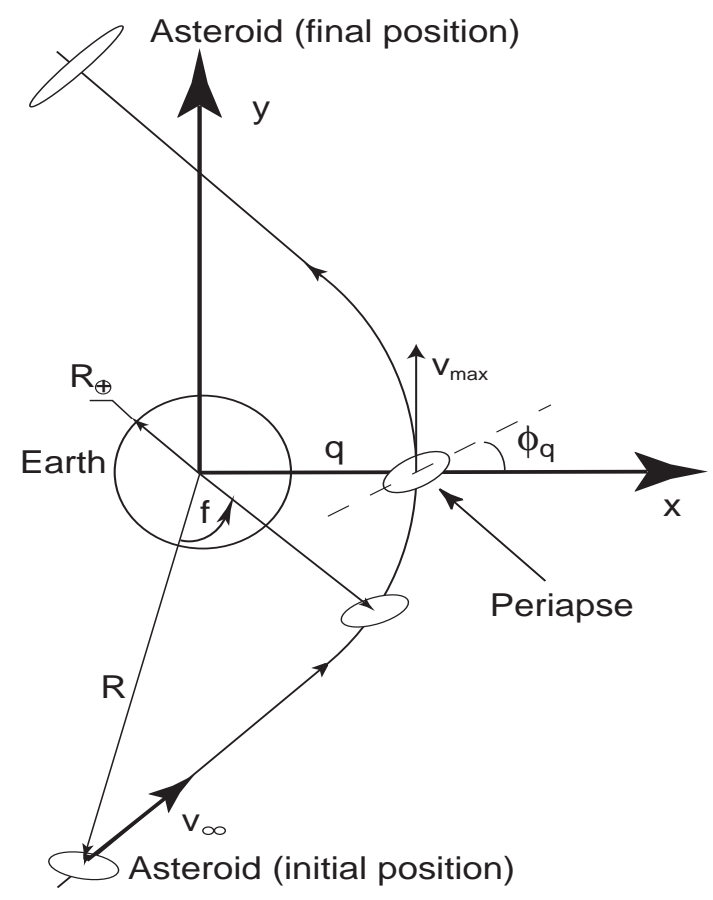

Fig. 1. Description of a fly-by orbit.

behavior.

We now specify the parameters governing an asteroid's planetary fly-by, where the planet is taken to be the Earth. To study a fly-by, we need first to specify the asteroid's orbit (its semi-major axis $a$ and eccentricity $e$ ). This is done by defining an encounter velocity $v_{\infty}$ (the velocity far away from the planet) and the distance of closest approach $q$. In our calculations, the asteroid starts at a distance, like the Moon's, of about sixty times the Earth's radius $R_{\oplus}$ to a comparable distance after its encounter. It is assumed that any changes in the asteroid's shape and spin state due to tidal interaction do not affect the orbit significantly, i.e., we ignore any spin-orbit coupling. Fig. 1 shows a schematic of the fly-by, where the problem is considered to be two-dimensional with the asteroid's spin normal to the orbit plane; we have chosen the initial conditions so that the pericenter lies on the $x$-axis. The asteroid is taken to be an ellipsoid with axes $a_{i}, i=1,2,3$ (see Sec. 5 below) that lie along principal axes in a coordinate system, indicated by the unit vectors $e_{1}$ and $e_{2}$ (with $e_{3}$ coming out of the paper); see Fig. 3.

We take the asteroid's initial shape to be a prolate ellipsoid with $a_{2} / a_{1}=\alpha=$ $\beta=a_{3} / a_{1}=0.6$, as opposed to the choice of Richardson et al. (1998), who used $\alpha=0.6$ and $\beta=0.55$. However, we do not expect this simplifying assumption to cause major differences from their results. The asteroid is considered to be spinning about the 3-axis. Richardson et al. (1998) also investigated the consequences of different initial spin periods and directions, and non-principalaxis rotations; the latter effect is not considered here, but we do remark upon 
the effect of the first two. However, the role of the long-axis' orientation at the time of the closest approach, and the effect of tidal torques in the vicinity of the periapse, are explored by varying the asteroid's semi-major axes' initial orientation with respect to the planet.

Other parameters are: $\rho_{A}$ - the asteroid's initial bulk density, $\rho_{s}$ - the mass density of the spheres assumed to comprise the aggregate (asteroid), $\rho_{\oplus}$ - the density of the Earth, $f(t)$ - the asteroid's angular position which varies with time, $R(t)$ - the asteroid's distance from the Earth, $\phi_{q}$ - the orientation of the asteroid's long axis at the time of closest approach, which is measured in an anti-clockwise direction from the periapse axis (see Fig. 1), and $m_{\oplus}$ and $m_{A}$ the masses of Earth and the asteroid, respectively.

It is also necessary to assume a description of the asteroid's interior, which we model as a rigid-granular material. Before the encounter we take the asteroid to be rigid. This assumption is consistent with the fact that present-day observations of asteroids in free motion show no shape changes. Furthermore, tightly packed aggregates show very little deformation, especially if the constituent "spheres" are hard enough and if the stresses due to body forces are compressive and do not change drastically (as might occur during a fly-by). In the granular state, the asteroid's mechanics are modelled by a constitutive law for loosely packed granular aggregates that is described later. We also utilize appropriate conditions to switch between the two states. Further on, we discuss the merits of modelling the granular aggregate (cf. Richardson et al. 1998) as a rigid-granular material.

As we will see below, because volume-averaged methods are used, the asteroid must always be an ellipsoid. Consequently, the model cannot describe breakup events although it can hint at their occurrence. The asteroid could be said to have broken apart if it becomes "too thin". As Sridhar and Tremaine (1992) point out, long thin ellipsoids are subject to instabilities due to their gravitational fields, which tend to tear them apart.

\section{Preliminaries}

We now describe how we will study this problem analytically, and lay out the mathematical formalism behind this approach. Those who wish to see only results should move directly to Sec. 6 .

In order to describe a rigid body's motion, it is enough to follow the evolution of the center-of-mass and the orientation of the body's principal inertia axes. However, for deformable bodies, we must take into account the evolution of each infinitesimal mass element. Fig. 2 shows the forces acting on one such 


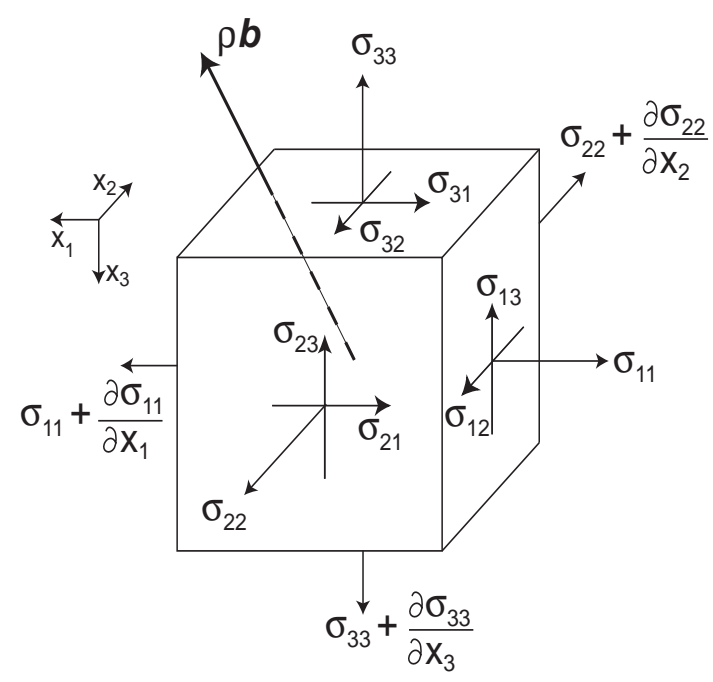

Fig. 2. Free body diagram of a typical mass element inside a body. Shear stresses on hidden faces are not shown, but they vary in a manner similar to the normal stresses.

mass element located at $\mathrm{x}$. Invoking force balance for this mass element, we obtain after dividing by the volume $d V$

$$
\sigma_{i j, j}+\rho b_{i}=\rho \ddot{x}_{i},
$$

in terms of the internal stresses $\sigma_{i j}$, the body force $\mathrm{b}$ and density $\rho$; this is the linear-momentum-balance equation. We use the indical notation and the summation convention throughout this paper, with each subscript ranging from one to three.

In the theory of elasticity, in order to determine the motion of a deformable body, it is necessary to solve for the stresses from the above equation while satisfying the prescribed boundary conditions, such as the surface of the body is free of applied forces. However, this in itself is not enough, as there are an infinity of solutions for the stresses that satisfy this equation (Fung 1965). In linear elasticity, the stresses unique to the solid under investigation are obtained by studying the displacement field associated with each stress solution. The displacements themselves are obtained by employing constitutive relations linking the stresses and the strains, e.g., a linear-elastic law or a Maxwellian visco-elastic material. In general, the associated displacement fields are not compatible. In order to obtain stresses that yield displacements that are compatible with the assumed material response of the body's interior, it is necessary that the stresses satisfy an extra set of equations, called the compatibility equations. The requirement of solving the compatibility equations makes the solution of elasticity problems extremely difficult. In the following, we will follow a volume-averaged approach, similar in spirit to St. Venant's semi-inverse method (Love 1946), where we assume the displacement field and solve for the accompanying stresses. Note that assuming a displacement field automatically 
satisfies the compatibility condition. Though approximate, this approach, as will be shown, can yield meaningful solutions in many contexts.

We now introduce some mathematical notation that will help in describing the volume-averaged approach later.

\subsection{Tensors or dyads}

In a coordinate system defined by the orthogonal unit vectors $\mathrm{e}_{i}$, a vector a can be written in terms of its components $a_{i}$ as

$$
\mathrm{a}=a_{i} \mathrm{e}_{i}
$$

Next, given two vectors a and $\mathrm{b}$, we define the tensor or dyadic product, denoted by $a \otimes b$, in terms of its components in the coordinate system formed by $\mathrm{e}_{i}$ above by

$$
(\mathrm{a} \otimes \mathrm{b})_{i j}=a_{i} b_{j}
$$

Higher-order products can be similarly defined. We note that the common operation of taking the cross-product of two vectors can be recovered by taking the vector associated with the anti-symmetric part of the tensor product of those two vectors.

We call the tensor product (or the dyadic product) of a vector field with a position vector, averaged over a space, as taking the first moment of that field. An immediate example is the inertia dyad $\boldsymbol{I}$ defined by

$$
I_{i j}=\int_{V} \rho x_{i} x_{j} d V
$$

which is obtained as the first moment of the mass distribution field $\rho \mathrm{x}$ in a body.

It is possible to take the first moment of each quantity in a vector equation. By assuming that infinitesimal mass elements of a rigid body interact only along their lines-of-center, we can "derive" Euler's equations for a rigid body by taking the anti-symmetric part of the volume-averaged, first moment of the linear momentum balance equation (1). This is illustrated below in Sec. 6. Taking the first and higher moments of a field, or an equation, that describes some characteristic of a system can be a powerful tool to obtain more information about the behavior of that system. 


\section{Governing equations: Volume-averaging}

We now provide some simple background on continuum modelling. Further details may be found in Fung (1965) or Holzapfel (2003). The number of dynamical equations required to describe a system depends on the degrees of freedom of that system. Thus, if we are interested in investigating the dynamics of a rigid body, we require six equations: three to follow the position of the center-of-mass and a trio more to follow the three Euler angles. Because it is composed of an infinite number of kinematically unconstrained points, a nonrigid body has infinite degrees of freedom. Thus, the governing equations are no longer ordinary differential equations but, instead, are a set of non-linear partial differential equations. As we noted above, solving these equations is very difficult analytically, and even numerical approaches are not easy. To obtain a more manageable theory, we need to reduce the degrees of freedom of a deformable body by putting restrictions on the displacement field.

A systematic procedure exists (Chadrasekhar 1969) by which theories for deformable bodies with increasing degrees of freedom can be formulated. The simplest such theory, and the one that we will employ here, assumes that the body's shape is a triaxial ellipsoid and that it can subsequently deform only into another ellipsoid. This deformation's description requires twelve kinematic variables: three to locate the center of mass of the ellipsoid, three Euler angles to locate the instantaneous principal axes of the ellipsoid, three stretches along the principal axes and three shears. Thus, this so-called homogeneous deformation has twelve degrees of freedom.

Once the center-of-mass is specified, the deformation of an ellipsoid into another ellipsoid can be described in terms of nine variables. This information can be encoded into a matrix $\boldsymbol{F}$, whose action on the position vector $\mathbf{X}$ of a mass element in the undeformed state yields the mass element's time dependent position vector $\mathrm{x}$ in the deformed state, i.e.,

$$
\mathrm{X}=\boldsymbol{F} \mathrm{X}
$$

The components of $\boldsymbol{F}$, which form the deformation gradient, consist of the nine time-dependent variables $F_{i j}(t)$ that, once known, would completely describe the evolution of the ellipsoid's shape.

To obtain a mass element's velocity, we differentiate the above equation with respect to time

$$
\dot{\mathrm{x}}=\dot{\boldsymbol{F}} \mathrm{X}
$$

this relates a mass element's velocity to its original position. It is useful to relate the mass element's velocity to its present position $\mathrm{x}$. This can be accomplished by utilizing the inverted form of (3) in the previous equation to 
obtain

$$
\dot{\mathrm{x}}=\dot{\boldsymbol{F}} \boldsymbol{F}^{-1} \mathrm{x},
$$

or

$$
\dot{x}=L x,
$$

with the definition

$$
\boldsymbol{L}=\dot{\boldsymbol{F}} \boldsymbol{F}^{-1} .
$$

Eqn. (4) relates a mass element's velocity to its present position, i.e., its position in the deformed ellipsoid. The components of $\boldsymbol{L}$ are the spatial gradients of the velocity in the deformed configuration, while $\boldsymbol{L}$ is called the velocity gradient. A useful decomposition of $\boldsymbol{L}$ is

$$
\boldsymbol{L}=\boldsymbol{D}+\boldsymbol{W}
$$

where $\boldsymbol{D}$ and $\boldsymbol{W}$ are $\boldsymbol{L}$ 's symmetric and anti-symmetric parts:

$$
\boldsymbol{D}=\operatorname{sym} \boldsymbol{L}=\frac{1}{2}\left(\boldsymbol{L}+\boldsymbol{L}^{T}\right)
$$

and

$$
\boldsymbol{W}=\operatorname{asym} \boldsymbol{L}=\frac{1}{2}\left(\boldsymbol{L}-\boldsymbol{L}^{T}\right)
$$

The quantity $\boldsymbol{D}$ is called the strain rate (or stretching rate) tensor while $\boldsymbol{W}$ is the local angular velocity (or spin) tensor. While $\boldsymbol{D}$ measures the rate at which the body is stretched and sheared, $\boldsymbol{W}$ measures the rate at which lines joining material points rotate. We employ the notation sym $\boldsymbol{B}$ and asym $\boldsymbol{B}$ to signify the operation of forming the symmetric and anti-symmetric parts of a tensor $\boldsymbol{B}$.

Finally, the mass element's acceleration is obtained by differentiating (4):

$$
\ddot{\mathrm{x}}=\dot{\boldsymbol{L}} \mathrm{x}+\boldsymbol{L} \dot{\mathrm{x}}=\left(\dot{\boldsymbol{L}}+\boldsymbol{L}^{2}\right) \mathrm{x} \equiv \boldsymbol{P} \times
$$

It now remains to obtain twelve ordinary differential equations to follow the evolution of these twelve kinematic variables. Three of these equations are given by force balance applied to the ellipsoid, which follows the acceleration of the center-of-mass. In our case, we need only two equations, as the fly-by occurs in a plane. The asteroid's center-of-mass is located by the angle $f(t)$ and its distance from the planet is $R(t)$ as shown in Fig. 1. The time evolution of these quantities can be expressed in terms of the orbital eccentricity $e$, the semimajor axis $a$ and the associated angular momentum $h_{\infty}\left(=\sqrt{G m_{P} a\left(1-e^{2}\right)}\right)$ of the hyperbolic orbit, which are in turn known once the encounter velocity $v_{\infty}$ and the distance at closest approach $q$ is specified. The evolution of the 
asteroid's angular position is (e.g., Murray and Dermott 1999 )

$$
\dot{f}=\frac{G^{2} m_{P}^{2}}{h_{\infty}^{3}}(1+e \cos f)^{2},
$$

while the asteroid's distance from the planet is

$$
R=\frac{a\left(1-e^{2}\right)}{1+e \cos f}
$$

The above equations completely specify the two-dimensional orbit of the asteroid during the fly-by.

The remaining nine equations are obtained by taking the first moment of the linear momentum balance equations (1) and averaging over the body's volume; hence, the term volume-averaging. Recall that the first moment was obtained by taking the tensor product of each term of Eqn. (1) with the position vector $x$ of the mass element, and averaging it over the body's volume:

$$
\int_{V} x_{k}\left\{\sigma_{i j, j}+\rho b_{i}=\rho \ddot{x}_{i}\right\} d V
$$

For the term on the right-hand side, we employ (9) to obtain

$$
\int_{V} \rho \ddot{x}_{i} x_{k} d V=\int_{V} \rho P_{i j} x_{j} x_{k} d V=P_{i j} \int_{V} \rho x_{j} x_{k} d V=P_{i j} I_{j k},
$$

where we have used the definition (2).

The second term on (12)'s left-hand side, i.e, the one involving the body force, is simply

$$
M_{k i} \equiv \int_{V} x_{k} \rho b_{i} d V
$$

with $M$ being called the moment tensor. Note, that the torque due to the external forces, which involves a cross-product between $\mathrm{x}$ and $\mathrm{b}$, can be obtained by taking the anti-symmetric part of $\boldsymbol{M}$.

Finally, (12)'s lead term that contains the divergence of the stress becomes

$$
\begin{aligned}
\int_{V} \sigma_{i j, j} x_{k} d V & =\int_{V}\left[\left(\sigma_{i j} x_{k}\right)_{, j}-\sigma_{i j} x_{k, j}\right] d V=\int_{S} \sigma_{i j} n_{j} x_{k} d S-\int_{V} \sigma_{i j} \delta_{j k} d V \\
& =\int_{S} T_{j} x_{k} d S-\int_{V} \sigma_{i k} d V
\end{aligned}
$$

where we have used Green's theorem to convert the first volume integral to a surface integral and have denoted $\sigma_{i j} n_{j}$ by $T_{j}$, which is simply the traction (force per unit area) on the ellipsoid's surface. In our applications, the asteroid's surface will be taken to be free from external forces. Thus, we drop the 
surface term from the previous equation. We also denote the volume-averaged stress $\int_{V} \boldsymbol{\sigma} d V$ by $\overline{\boldsymbol{\sigma}} V$, where $\overline{\boldsymbol{\sigma}}$ denotes the average stress and $V$ the ellipsoid's volume, and rewrite the previous equation by

$$
\int_{V} \sigma_{i j, j} x_{k} d V=-\bar{\sigma}_{i k} V
$$

When a body's allowed deformations are restricted to homogeneous deformations (i.e., ellipsoids deforming only into ellipsoids), the stresses within it are a constant. This is because constitutive laws relate the stress to the deformation gradient $\boldsymbol{F}$, which is a constant through the body. Thus, we subsequently drop the overline on $\boldsymbol{\sigma}$.

Finally, we bring together the results from Eqns. (13-15) into (12) to obtain

$$
P_{i j} I_{j k}=M_{k i}-\sigma_{i k} V
$$

which on reverting to more direct notation, substituting back for $\boldsymbol{P}$ from (9) and taking the equation's transpose, becomes

$$
\left(\dot{\boldsymbol{L}}+\boldsymbol{L}^{2}\right) \boldsymbol{I}=\boldsymbol{M}^{T}-\boldsymbol{\sigma} V
$$

The above equation is simply a balance, in a volume-averaged sense, between the inertial forces represented by the left-hand side, the body force and the resistance to deformation of the body. Because the body's shape may change, so too may its inertia dyad $\boldsymbol{I}$. Thus, in order to complete the dynamical description of a deforming ellipsoid, it is necessary to provide an additional equation for the inertia dyad. This equation can be obtained directly by differentiating (2):

$$
\begin{aligned}
\dot{I}_{i j} & =\int_{V} \rho \dot{x}_{i} x_{j} d V+\int_{V} \rho x_{i} \dot{x}_{j} d V=\int_{V} \rho L_{i k} x_{k} x_{j} d V+\int_{V} \rho x_{i} L_{j k} x_{k} d V \\
& =L_{i k} \int_{V} \rho x_{k} x_{j} d V+L_{j k} \int_{V} \rho x_{i} x_{k} d V=L_{i k} I_{k j}+L_{j k} I_{i k},
\end{aligned}
$$

which, when written in an index-free notation, becomes

$$
\dot{\boldsymbol{I}}=\boldsymbol{L} \boldsymbol{I}+\boldsymbol{I} \boldsymbol{L}^{T}
$$

Eqns. 16 and 17 will be used to solve for the dynamical evolution of the deforming ellipsoid (within the assumed restrictions on the deformation), once the effects of external forces as captured by $\boldsymbol{M}$ and a constitutive law relating the material response $\boldsymbol{\sigma}$ to the deformation $\boldsymbol{L}$ are prescribed. 


\section{Material behavior}

In order to use the volume-averaged equation (16), it is necessary to have a model for the asteroid's material behavior. As we mentioned above, we consider the asteroid to be a rigid-granular material, which is rigid until one of two transition criteria (introduced below) governing the material's passage from a rigid to a granular state is violated. When the body is in a rigid state, its material behavior is simple enough; however, its response (i.e., the relation between stress and strain, or its constitutive law) in a granular state is complicated, as outlined below. We also specify when the material transitions from a granular state back into a rigid state.

\subsection{A continuum law for the granular state}

This continuum model attempts to capture the essential features of the material response of a loose collection of inelastic spheres with radius $r$, coefficient of restitution $\epsilon$ and mass density $\rho_{s}$. Many flows of granular materials are modelled by such collections of spheres (e.g., Jenkins and Savage 1983). Although Jenkins and Savage (1983) took the spheres to be smooth, we could allow them to be frictional if the coefficient of sliding friction were small, say less than 0.2. In this case, the stresses are unaffected and the energy losses due to friction can be accounted for in the coefficient of restitution (Jenkins and Zhang 2002).

The motivation for using this description to model asteroids comes from the work of Richardson et al. (1998) and several others. The asteroid was modeled as a collection of smooth inelastic spheres that moved about colliding with each other, with internal self-gravity being the confining force. Inelasticity was included through the coefficient of restitution being less than unity. In such an approach, the motion of each particle was followed and no attempt was made to develop, or use, a continuum model for the asteroid. A primary aim in studying the mechanics of loose granular aggregates is to test the hypothesis that asteroids might actually have interiors similar to these.

The derivation of a continuum model for such a system invokes the obvious analogy between colliding spheres and interacting molecules of a dense gas (Jenkins and Savage 1983). This analogy is further reinforced by introducing the notions of granular pressure and granular temperature, whose definitions carry over directly from kinetic theory with molecules replaced by spheres. Thus, granular pressure, as measured by a confining surface, would be the mean time-rate of momentum transfer per unit area of the surface due to impacts of the spheres. Similarly, granular temperature measures the mean 
translatory kinetic energy of the spheres as seen in a frame translating with the mass motion of the aggregate. Thus, this estimates the kinetic energy hidden in the fluctuations of the spheres alone. If we assume that the coefficient of sliding friction is small, the exchange of angular momentum is small and the rotational kinetic energy can be simply related to the translational kinetic energy (Jenkins and Zhang 2002).

Methods of the kinetic theory of dense gases are used to derive the constitutive law for this loose aggregate. Analogous to dense gases (Chapman and Cowling 1970), we obtain that the stress in a granular material is related to the deformation rate by

$$
\boldsymbol{\sigma}=\left(-p^{\prime}+\varpi D_{k k}\right) \boldsymbol{1}+2 \eta \boldsymbol{D},
$$

where $\mathbf{1}$ is the identity matrix, $p^{\prime}$ is the previously defined granular pressure, $\varpi$ the bulk viscosity and $\eta$ the shear viscosity. The constitutive relation above is similar to that of a compressible, Newtonian fluid. The finite-size effect of the constituent spheres enters via the three quantities $p^{\prime}, \varpi$ and $\eta$ as listed directly below. These can be related to the granular temperature $T$ and the aggregate's density $\rho$ via

$$
\begin{aligned}
& p^{\prime}=\rho(1+4 \hat{g}) T, \\
& \varpi=\frac{8}{3 \sqrt{\pi}} \rho r \sqrt{T} \hat{g}
\end{aligned}
$$

and

$$
\eta=\frac{\sqrt{\pi}}{6} \rho r \sqrt{T}\left[\frac{5}{16 \hat{g}}+1+\frac{4}{5}\left(1+\frac{12}{\pi}\right) \hat{g}\right],
$$

with the factor $\hat{g}$ given by

$$
\hat{g}(\nu)=\nu g_{0}(\nu)
$$

where $\nu$ is the solid volume fraction, which is the ratio of the aggregate's density to the density of its constituent spheres, and $g_{0}(\nu)$ incorporates the influence of the volume occupied by the spheres on their collisional frequency. In terms of $\nu$, it is (Jenkins and Savage 1983; cf. Torquato 1995):

$$
g_{0}(\nu)=\left\{\begin{array}{cc}
\frac{2-\nu}{2(1-\nu)^{3}}, & 0.01 \leq \nu \leq 0.5, \\
\frac{3(0.64-0.5)}{\nu(0.64-\nu)}, & 0.5 \leq \nu<0.64 .
\end{array}\right.
$$

The above formula indicates that a sphere's collisional frequency increases to infinity as $\nu$ approaches 0.64 - the volume fraction of a random, close packed aggregate of identical spheres (Torquato 2001). This models the decrease in a sphere's mean free path as the granular medium becomes denser. 
In order to be able to use (18), we need an equation for $T$ 's evolution. To this end, energy balance is used to yield

$$
\frac{3}{2} \rho \dot{T}=-q_{k, k}+\sigma_{i j} D_{i j}-\gamma
$$

Thus, the time-rate of change in fluctuation energy (as measured by the change in temperature) is balanced by the divergence of the flux of fluctuation energy q, the work done by the stresses $\left(\sigma_{i j} D_{i j}\right)$ and the collisional rate of dissipation of fluctuation energy per unit volume $\gamma$. This equation is very similar to the classical one obtained for gases (Chapman and Cowling 1970), except for the presence of the non-classical dissipation term $\gamma$. Due to this dissipation, steady states are possible in cases where they were not for an aggregate of elastic spheres. The parameter $\gamma$ can be shown (Jenkins and Savage 1983) to be

$$
\gamma=\frac{24}{\sqrt{\pi}}(1-\epsilon) \frac{\rho T^{\frac{3}{2}}}{r} \hat{g}
$$

After this short summary of the theory, we derive the constitutive model to be used for further analysis. Note that though these are loose aggregates, the volume fraction is still high $(\nu \geq 0.4)$, so that $\hat{g} \geq 1.5$. This allows us to approximate (19) as

$$
p^{\prime} \approx 4 \rho \hat{g} T
$$

and (21) as

$$
\eta \approx \frac{8}{5 \sqrt{\pi}}\left(1+\frac{\pi}{12}\right) \rho r \sqrt{T} \hat{g}
$$

Comparing the above expression with (20), we see that the bulk and shear viscosities are now very simply related:

$$
\eta \approx 0.76 \varpi
$$

Next, we simplify (24) by assuming that the rate of work done by the stresses is balanced by the collisional dissipation of fluctuation energy $\gamma$, i.e.,

$$
\sigma_{i j} D_{i j}-\gamma=0
$$

This assumption says that the temperature $T$ reaches equilibrium on a time scale much faster than the dynamic time scale. It also assumes that the term $q_{k, k}$ is zero, which is a reasonable assumption to make if we believe that the amount of energy lost from the asteroid's surface is small. Now, using the expressions for $\gamma$ from (25) and $\boldsymbol{\sigma}$ from (18) in the equation above, we obtain the granular temperature $T$ in terms of the strain rate:

$$
T=\frac{r D_{k k} \sqrt{\pi}}{12(1-\epsilon)}\left[-1+\left\{1+\frac{16}{\pi}(1-\epsilon)\left(1+\frac{6}{5}\left(1+\frac{\pi}{12}\right) \frac{D_{i j}^{\prime} D_{i j}^{\prime}}{D_{k k}^{2}}\right)\right\}^{\frac{1}{2}}\right]
$$


with $\boldsymbol{D}^{\prime}$ defined in terms of the total deformation rate $\boldsymbol{D}$ by

$$
D_{i j}^{\prime}=D_{i j}-\frac{1}{3} D_{k k} \delta_{i j}
$$

and is a measure of the shear rate, as the volumetric change $D_{k k} / 3$ has been subtracted.

The assumption of quasi-static growth in $T$ is not really crucial to further development, but it does reduce the number of variables by one, because now $T$ is known explicitly in terms of $\boldsymbol{D}^{\prime}$. We can, if required, keep (24) as a separate equation governing the time evolution of $T$, but no significant effect on the results is expected, so we prefer to work with (29).

Eqns. (18), (20), (26), (27) and (29), with $\hat{g}$ defined through $g_{0}$ via (22) and (23), give a complete description of the material response for a collection of inelastic spheres. From these equations, we notice that the stress $\boldsymbol{\sigma}$ can be expressed as only a function of the components of the deformation rate $\boldsymbol{D}$. We use this function in (16) along with expressions for the moment tensor $\boldsymbol{M}$ derived below, to obtain an equation in terms of kinematic variables alone. Recall that $\boldsymbol{D}$ and $\boldsymbol{L}$ are related by (6).

\subsection{Transition between rigid and granular states}

Assuming that the asteroid is initially rigid has consequences on the body's subsequent deformation, as the asteroid cannot change shape until it transitions to a granular state. This contrasts with the model of Richardson et al. (1998) and, as discussed later, significantly affects the asteroid's overall behavior. Several failure criteria that govern this transition can be postulated. For example, the transition might occur when the average pressure in the asteroid's interior becomes negative. However, this is too restrictive, as a single principal stress may become tensile even though the overall pressure remains compressive. So, because granular materials are unable to sustain tensile loads, a better failure law would be (Tensile Criterion) that the transition occurs whenever any principal stress becomes positive. This global criterion differs from the one that is inherently built into Richardson et al.'s (1998) model. Under their computational scheme, the body sheds mass when the net force on a sphere, on the body's surface, ceases to be attractive. This can occur even while the average principal stresses in the body are negative (compressive). Another possible failure law (Mohr-Coulomb) considers the aggregate to transition from a rigid to a granular state whenever it violates the Mohr-Coulomb criterion, which is commonly used to predict failure in soils. According to this criterion, the transition occurs when the maximum shearing stress at a point reaches a critical value that is a function of the normal stress on that same 
plane, i.e., when the inequality

$$
\sigma_{\max }-k_{M C} \sigma_{\min } \leq 0
$$

is violated. In the above, $\sigma_{\max }$ and $\sigma_{\min }$ are the maximum and minimum principal compressive stresses. The parameter $k_{M C}$ is related to the internal friction angle $\phi_{F}$ by

$$
k_{M C}=\frac{1+\sin \phi_{F}}{1-\sin \phi_{F}} .
$$

By examining Mohr's circle, which relates shear and normal stresses across planes with different orientations, it is easily seen that the tensile criterion results in a later failure than the Mohr-Coulomb criterion (e.g., Lambe and Whitman 1969 or Chen and Han 1988).

We define the asteroid's transition from a granular to a rigid state in a simple way. The asteroid is considered to be rigid whenever the volume fraction $\nu$ in the granular state increases above a critical cut-off, which we take to be 0.639 . This number is chosen because it is close to 0.64 , which recall was the volume fraction of a random, close-packed aggregate of identical spheres. The cut-off is not set precisely at a $\nu$ of 0.64 to avoid the singularity inherent in Eqn. (23). As noted later, such a transition criterion tends to hinder disruption because, according to it, asteroids pass into a rigid state rather easily.

\section{Moments}

We now consider both the moment tensor due to self-gravity and the quadrupole moment due to the planet's gravitational field. These expressions will be used in the following section.

\subsection{Gravity moment tensor $\boldsymbol{M}_{\boldsymbol{G}}$}

Consider a coordinate system with its origin at the ellipsoid's center-of-mass and defined by unit vectors $\hat{e}_{i}$ along the ellipsoid's semi-major axes (see Fig. 3). The gravitational potential at a point $\mathrm{x}=x_{i} \hat{\mathrm{e}}_{i}$ inside a triaxial ellipsoid is (Kellogg 1953 or Chandrasekhar 1969)

$$
U=-\pi \rho G\left(A_{1} x_{1}^{2}+A_{2} x_{2}^{2}+A_{3} x_{3}^{2}\right),
$$

where we omit the additive constant as it has no effect on the body force. The quantities $A_{i}$ depend on the geometry (i.e., the semi-major axes $a_{i}$ ) alone. 
Chandrasekhar (1969) notes an important property:

$$
\sum_{i=1}^{3} A_{i}=2
$$

Thus, for a sphere, $A_{1}=A_{2}=A_{3}=2 / 3$. In general it is sufficient to know two of the $A_{i}$ 's, with the third being calculated from (33). For a prolate ellipsoid, where $1>\alpha=\beta$,

$$
A_{3}=A_{2}=\frac{1}{\sqrt{1-\beta^{2}}}-\frac{\beta^{2}}{2\left(1-\beta^{2}\right)^{3 / 2}} \ln \frac{1+\sqrt{1-\beta^{2}}}{1-\sqrt{1-\beta^{2}}},
$$

while by employing (33),

$$
A_{1}=2\left(1-A_{3}\right) \text {. }
$$

In the given coordinate system we define the shape tensor $\boldsymbol{A}$ as

$$
A_{i j}=\left\{\begin{array}{cc}
A_{i} & i=j \\
0 & i \neq j
\end{array}\right.
$$

This tensor captures the effect of the body's shape on its internal gravitational field. The potential $U$ can now be rewritten as

$$
U=-\pi \rho G A_{i j} x_{i} x_{j}
$$

The force acting on a mass element $d m$ at $\mathrm{x}$ is simply $\nabla U$. Then the body force due to self-gravity is

$$
\begin{aligned}
\mathrm{b}_{\mathrm{G}} & =-2 \pi \rho G\left(A_{1} x_{1} \hat{\mathrm{e}}_{1}+A_{2} x_{2} \hat{\mathrm{e}}_{2}+A_{3} x_{3} \hat{\mathrm{e}}_{3}\right) \\
& =-2 \pi \rho G \boldsymbol{A} \mathbf{x} .
\end{aligned}
$$

Using this in (14) and recognizing that $\boldsymbol{A}$ is a constant, the gravity moment tensor $\boldsymbol{M}_{\boldsymbol{G}}$ is

$$
\boldsymbol{M}_{\boldsymbol{G}}=-2 \pi \rho G \boldsymbol{I} \boldsymbol{A} .
$$

Note that the principal axes of $\boldsymbol{I}$ and $\boldsymbol{A}$ are the same and coincide with the principal axes of the deformed ellipsoid. Thus, $\boldsymbol{I}$ and $\boldsymbol{A}$ commute.

\subsection{Quadrupole moment tensor}

The quadrupole moment tensor $\boldsymbol{M}_{Q}$ is generated by the forces exerted by an external body of mass $m_{P}$. In order to calculate $\boldsymbol{M}_{\boldsymbol{Q}}$, consider Fig. 3 , where

$$
D^{2}=r^{2}+R^{2}-2 r R \cos \theta
$$




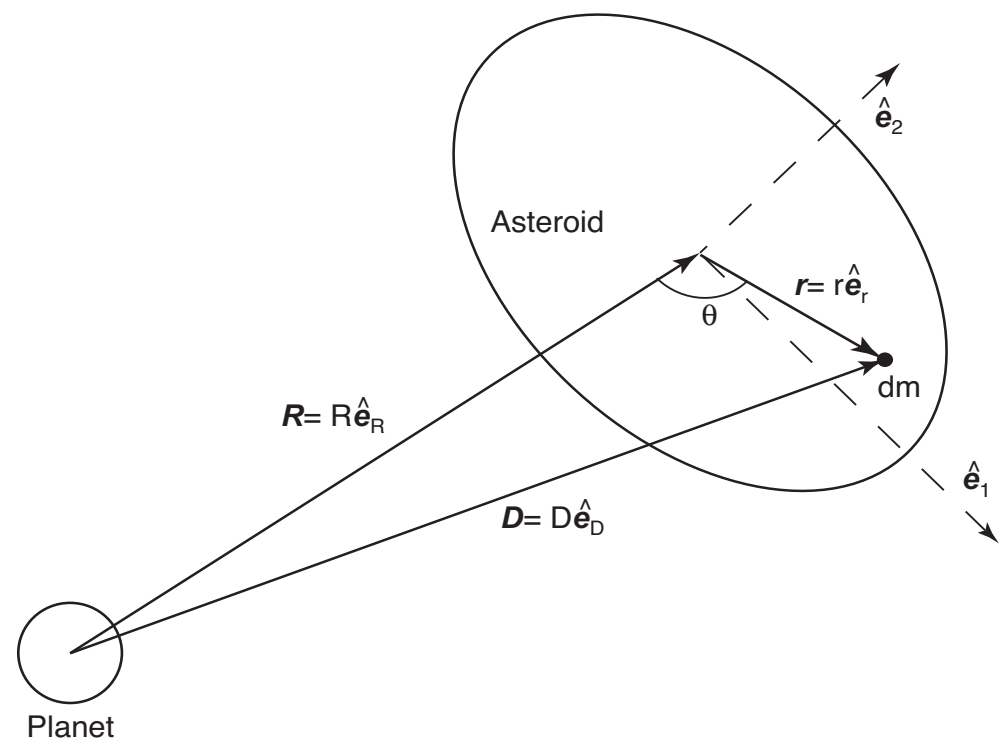

Fig. 3. Figure showing the geometry used to calculate the quadrupole moment $\boldsymbol{M}_{Q}$.

and

$$
D \hat{\mathrm{e}}_{D}=R \hat{\mathrm{e}}_{R}+r \hat{\mathrm{e}}_{r} .
$$

The body force on a unit mass (i.e., the acceleration) is

$$
\mathrm{b}_{Q}=\frac{d \mathrm{~F}}{d m}=-G \frac{m_{P}}{D^{2}} \hat{\mathrm{e}}_{D}=-\frac{G m_{P} R}{D^{3}}\left(\hat{\mathrm{e}}_{R}+\frac{r}{R} \hat{\mathrm{e}}_{r}\right) .
$$

We use this in (14) to obtain

$$
M_{Q}=-\int_{V} \frac{\rho G m_{P} R}{D^{3}} r\left(\hat{\mathrm{e}}_{r} \otimes \hat{\mathrm{e}}_{R}+\frac{r}{R} \hat{\mathrm{e}}_{r} \otimes \hat{\mathrm{e}}_{r}\right) d V
$$

Recall from Sec. 2 that $\hat{\mathrm{e}}_{r} \otimes \hat{\mathrm{e}}_{r}$ denotes the tensor product of $\hat{\mathrm{e}}_{r}$ and $\hat{\mathrm{e}}_{r}$. In indical notation it is simply the matrix $\hat{e}_{r_{i}} \hat{e}_{r_{j}}$, where the components are evaluated in the coordinate system shown in Fig. (3).

For most applications, such as planetary fly-bys, $r \ll R$. Expanding $1 / D^{3}$ in $r / R$ and retaining terms of order $r^{2} / R^{2}$, we find

$$
\frac{1}{D^{3}} \sim \frac{1}{R^{3}}\left[1+3 \cos \theta \frac{r}{R}+\frac{3}{2}\left(5 \cos ^{2} \theta-1\right) \frac{r^{2}}{R^{2}}\right] .
$$

Substituting this into (42) and keeping terms of order $r^{2} / R^{2}$ yields

$$
\begin{array}{r}
M_{Q} \approx-\frac{G m_{P}}{R^{3}}\left[\int_{V} \rho r R\{1+3 \cos \theta\right. \\
\left.\frac{r}{R}+\frac{3}{2}\left(5 \cos ^{2} \theta-1\right) \frac{r^{2}}{R^{2}}\right\}\left(\hat{\mathrm{e}}_{r} \otimes \hat{\mathrm{e}}_{R}\right) d V \\
\left.+\int_{V} r^{2}\left(1+3 \cos \theta \frac{r}{R}\right)\left(\hat{\mathrm{e}}_{r} \otimes \hat{\mathrm{e}}_{r}\right) d V\right]
\end{array}
$$


To evaluate the various integrals above, we use the center-of-mass's definition and symmetry arguments to find

$$
\begin{gathered}
\int_{V} r \hat{\mathrm{e}}_{r} \otimes \hat{\mathrm{e}}_{R} d V=0, \quad \int_{V} r^{3} \hat{\mathrm{e}}_{r} \otimes \hat{\mathrm{e}}_{R} d V=0 \\
\int_{V} r^{3} \cos ^{2} \theta \hat{\mathrm{e}}_{r} \otimes \hat{\mathrm{e}}_{R} d V=0 \quad \text { and } \quad \int_{V} r^{3} \cos \theta \hat{\mathrm{e}}_{r} \otimes \hat{\mathrm{e}}_{r} d V=0 .
\end{gathered}
$$

Additionally,

$$
\int_{V} r^{2} \cos \theta \hat{\mathrm{e}}_{r} \otimes \hat{\mathrm{e}}_{R} d V=-\boldsymbol{I} \hat{\mathrm{e}}_{R} \otimes \hat{\mathrm{e}}_{R}
$$

and

$$
\int_{V} r^{2} \hat{\mathrm{e}}_{r} \otimes \hat{\mathrm{e}}_{r} d V=\boldsymbol{I}
$$

where the latter follows directly from (2) and the former is obtained by using $\cos \theta=\hat{\mathrm{e}}_{r} \cdot \hat{\mathrm{e}}_{R}$. Thus,

$$
\boldsymbol{M}_{\boldsymbol{Q}}=-\frac{G m_{P}}{R^{3}} \boldsymbol{I}\left(\boldsymbol{1}-3 \hat{\mathrm{e}}_{R} \otimes \hat{\mathrm{e}}_{R}\right)
$$

or,

$$
M_{Q_{i j}}=-\frac{G m_{P}}{R^{5}} I_{i k}\left(R^{2} \delta_{k j}-3 R_{k} R_{j}\right) .
$$

These provide the quadrupole moment tensor in terms of the position of the external perturber, its mass, and the inertia dyad (tensor) of the body itself. Note that the skew part of $\boldsymbol{M}_{\boldsymbol{Q}}$, which represents the quadrupole torque, agrees with MacCullagh's formula (see, e.g., Murray and Dermott 1999, pp. 197-198). Furthermore, our expression for $\boldsymbol{M}_{\boldsymbol{Q}}$ agrees with that of Sridhar and Tremaine (1992), providing a further check.

\section{Results}

We now summarize how we will investigate planetary fly-bys of asteroids that are modeled as rigid-granular prolate ellipsoids. In order to follow an encounter we need to know the position of the asteroid's center-of-mass as well as its shape, which may change during the fly-by. The asteroid, i.e., its center-ofmass, is required to fly along an orbit that is determined by choosing its initial distance from the planet, the encounter velocity $v_{\infty}$, and the distance at closest approach $q$. The location of the asteroid's center-of-mass is obtained from (10) and (11), which are integrated numerically.

The asteroid's change in rotation rate and possible distortion in shape are obtained by judicious use of (16). At the beginning of the encounter, the asteroid is taken to be rigid, so that $\boldsymbol{I}$ is constant. The rotational dynamics then is described completely by the spin tensor $\boldsymbol{W}$, which, in the case of a rigid body, is the same as the anti-symmetric tensor associated with the 
angular velocity measuring the rotation rate of the ellipsoid's principal axes. The equation for $\boldsymbol{W}$ 's evolution is obtained by noting that because there is no change in shape, $\boldsymbol{D}=0$ and $\boldsymbol{L}=\boldsymbol{W}$, so that taking the anti-symmetric part of (16) yields

$$
\operatorname{asym}\left[\left(\dot{\boldsymbol{W}}+\boldsymbol{W}^{2}\right) \boldsymbol{I}\right]=\operatorname{asym} \boldsymbol{M}^{T},
$$

where recall that the notation asym denotes the anti-symmetric part of a tensor. Note that (44) is really three equations, which are, as was to be expected, the Euler equations for a rigid body, with the right-hand side being the tidal torque given by the anti-symmetric part of the quadrupole moment tensor $\boldsymbol{M}_{Q}$ from (43). The internal gravity, being radial (i.e., has zero curl), makes no contribution to the rigid-body torque.

As the asteroid increasingly feels the planet's gravitation, it may start to disrupt. This can occur when either of the two failure criteria discussed previously is violated. We explore the two criteria separately below. In general, for each choice, we use the principal stresses of the average rigid-body stress tensor at every integration step to check whether or not the chosen failure criterion is violated. The rigid-body stress tensor is obtained by taking the symmetric part (denoted by sym) of (16)

$$
\boldsymbol{\sigma}=\frac{1}{V} \operatorname{sym}\left[\boldsymbol{M}^{T}-\left(\dot{\boldsymbol{W}}+\boldsymbol{W}^{2}\right) \boldsymbol{I}\right],
$$

where the moment tensor now includes contributions from both the quadrupole moment tensor and the gravity moment tensor $\boldsymbol{M}_{\boldsymbol{G}}$ given by (39). If the chosen disruption criterion has indeed been violated, we assume that the asteroid has begun to disrupt and its interior is then modelled by a granular rheology. As the asteroid's shape can now change, $\boldsymbol{D} \neq 0$, and we have to solve for all nine components of $\boldsymbol{L}$ from (16), along with the six components of the inertia dyad $\boldsymbol{I}$ from (17). This is easily done numerically as it involves the integration of a coupled set of ordinary differential equations, once we have substituted for the stress tensor $\boldsymbol{\sigma}$ in (16) by employing (18), (20), (26), (27) and (29) to relate the stress tensor to the deformation rate $\boldsymbol{L}$, and have used the formulae for the gravity moment tensor from (39) and the quadrupole moment tensor from (43) to calculate the total moment tensor $\boldsymbol{M}$. At this time, the motion of the asteroid's center-of-mass is still assumed to follow the previously determined orbit.

Post-planetary encounter, a disrupted asteroid can re-accumulate. This will occur, as was outlined earlier, when the volume fraction of the asteroid becomes large enough. We check this by calculating the volume fraction at each integration step, using the constancy of mass of the asteroid and its deformed shape, which in turn is found using the changing inertia dyad $\boldsymbol{I}$. Recall that in our model the ellipsoid can deform, but does not shed mass. Once the as- 
teroid re-accumulates and become rigid, its evolution is again easily followed. Note that during disruption the asteroid's volume changes. Though any constituent sphere's density remains unchanged, constancy of mass is enforced by a varying volume fraction.

From the paragraphs above, we see that once we choose a transition criterion from the two at our disposal, we have a closed set of ordinary differential equations to model the asteroid's fly-by. The evolution of the asteroid's size, as described by the semi-major axes of its ellipsoid, indicates the outcome of that fly-by. Following Richardson et al. (1998), we choose the coefficient of restitution $\epsilon$ that governs the energy dissipation in our continuum model to be 0.8 , the asteroid's initial bulk density $\rho_{A}$ is taken to be $2 \mathrm{~g} / \mathrm{cm}^{3}$, and the constituent sphere's density $\rho_{s}$ to be $3.6 \mathrm{~g} / \mathrm{cm}^{3}$; the latter choices correspond to an initial volume fraction of $\nu=\rho_{A} / \rho_{s}=0.56$. Finally, we consider three different spin periods and look at both prograde and retrograde rotations.

Our numerical integration was carried out using the built-in ODE solver ode45 in MATLAB. Some limiting tests were carried out to validate the code. For example, when we set the planetary mass to zero and allowed the asteroid to execute full three-dimensional rotation, we obtained a linear path for the asteroid's center-of-mass, and free rigid-body nutational motion in which angular momentum and energy were conserved, as was to be expected. Similarly, if the asteroid's shape was taken to be spherical, angular momentum and rotational energy were conserved and we recovered the solution for a point mass flying past a planet.

\subsection{Outcomes with the tensile criterion}

We first investigate the behavior of a rigid-granular asteroid whose disruption is controlled by the tensile criterion described in Sec. 4.2. We choose an initially rigid asteroid in prograde rotation, with axes ratios $\alpha=\beta=0.6$, located at a distance of around $60 R_{\oplus}$ from the planet, and follow the solution strategy outlined in the previous section.

Fig. 4 tabulates the outcomes of fly-bys as the encounter velocity $v_{\infty}$ and distance at closest approach $q$ are varied. The asteroid's initial spin period was taken to be six hours. For each fly-by, several initial orientations of the asteroid, which lead to different long-axis orientations $\phi_{q}$ at the point of closest approach (periapse), were considered. The table uses notation similar to that of Richardson et al. (1998), viz.,

- S: if the encounter results in a significant amount of deformation. The encounter outcome could potentially be a Shoemaker-Levy-9-type string of material (Asphaug and Benz 1996). An outcome where any one of the final 


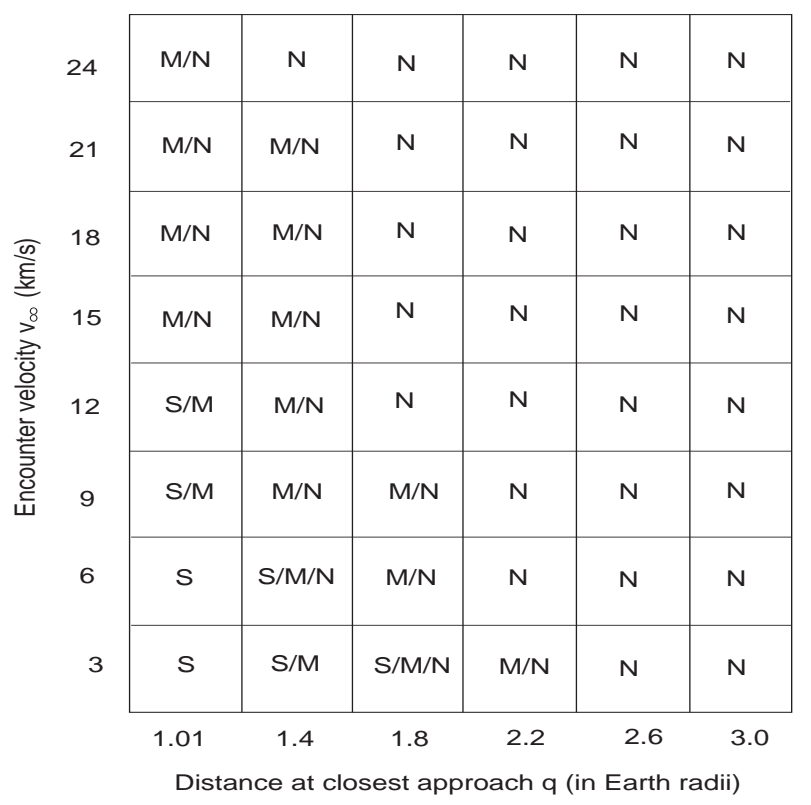

Fig. 4. The outcomes of Earth fly-bys are tabulated for various encounter velocities $v_{\infty}$ and distances of closest approach $q$. The asteroid's initial prograde spin period was six hours. The tensile criterion is used to model the transition from a rigid to a granular state. The symbols $\mathrm{S}, \mathrm{M}$ and $\mathrm{N}$ denote, respectively, fly-bys leading to $S$-L-9 catastrophic break-ups, fly-bys where major shape changes take place, and fly-bys where essentially no shape change occurs. Multiple entries, e.g., M/N, are defined in the text. See the discussion in Sec. 6.3.

axes ratios is less than 0.3 is classified as an S-type.

- M: when the asteroid is not broken apart, but does suffer major change in shape. Outcomes where the final axes ratios lie between 0.3 and 0.5 are classified as M-types.

- N: when the asteroid's shape has not changed appreciably at the end of the fly-by. The final axes ratios lie between 0.5 and 0.6.

As mentioned earlier, because of the model's limitations, the asteroid cannot be seen to separate physically. So even though we can, with sufficient confidence, predict whether an asteroid will break apart or not, it is impossible to predict whether the progeny will be a binary or a string of objects. Thus, all break-up events are marked by an $\mathrm{S}$ in Fig. 4. The outcomes identified by $\mathrm{S} / \mathrm{M} / \mathrm{N}$ (and similarly for $\mathrm{M} / \mathrm{N}$ ) denote cases where the asteroid can be broken apart, reshaped, or not affected, depending on the orientation of the long-axis at periapse. This is because the outcome of a fly-by relies crucially on the interactions near periapse, as the magnitude of the planet's quadrupole moment, and changes in spin due to planetary tidal torque, differ with the orientation of the long-axis near the periapse.

Before proceeding to analyze the results tabulated in Fig. 4, we make some prefatory remarks. For an asteroid to undergo disruption, failure has to be 


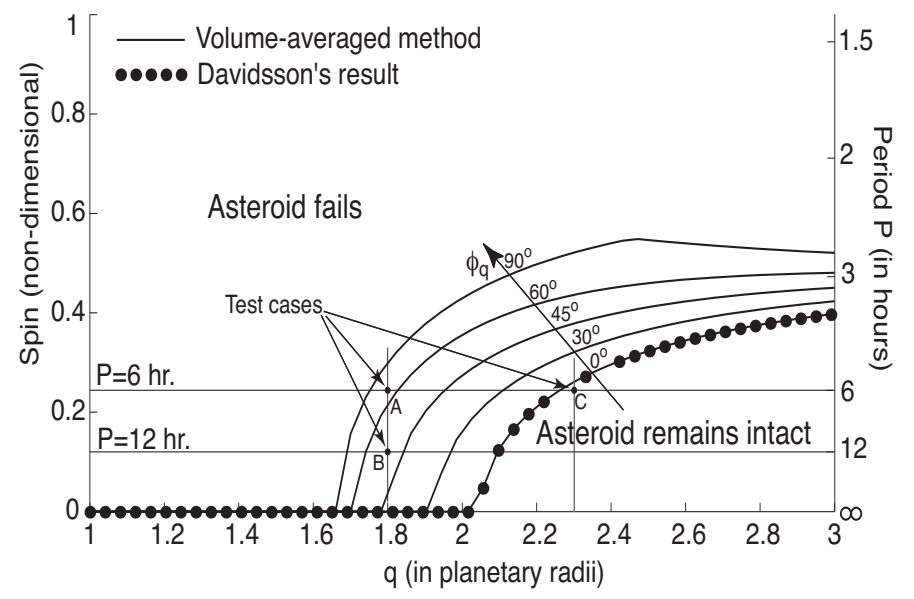

Fig. 5. The Roche limit with respect to the Earth for a prolate ellipsoid with axes ratios $\alpha=\beta=0.6$ as a function of the long-axis orientation $\phi_{q}$ and its spin, as calculated by the volume-averaged method. Davidsson's (2001) result for $\phi_{q}=0^{\circ}$ is also shown for comparison. The test cases are discussed in Sec. 6.3.

initiated, i.e., at some point during its fly-by, the stresses in the asteroid must satisfy conditions for failure, which in the present case (Tensile failure criterion) amounts to one principal stress becoming positive (tensile). After failure is initiated, the rheology is no longer a rigid one, but granular, which permits deformation. Once failure occurs, subsequent disruption depends on the magnitude of the quadrupole moment $\boldsymbol{M}_{\boldsymbol{Q}}$, the gravitational moment $\boldsymbol{M}_{\boldsymbol{G}}$, and also on changes in spin due to both tidal torques and the asteroid's changing shape.

In order to probe the mechanics of tidal encounters, two different initial spin periods (either 6 or 12 hours) were employed. We summarize the effects of different initial spins in Sec. 6.5. Further, two different periapse distances of $1.8 R_{\oplus}$ and $2.3 R_{\oplus}$ were used. In the following, we have non-dimensionalized the time by $1 / \sqrt{2 \pi \rho_{s} G}$, which is approximately 0.23 hours, corresponding to scaling the spin rate by 0.7 rotations/hour.

\subsection{The Roche limit}

To better understand the disruption process it is worthwhile to consider the Roche limit of a granular ellipsoidal body. The classical Roche limit is the distance from a planet within which a homogeneous, synchronously rotating, fluid body will disrupt; it is 2.46 times the planetary radius for bodies of equal densities (Murray and Dermott 1999, pp. 158-159). It is obtained by balancing the stabilizing effects of the body's self-gravity against the disruptive effects of planetary tides and the centrifugal "force" due to the body's spin (Chandrasekhar 1969). In fact, for a real object this distance depends on, 
amongst other things, the body's rotation rate, its and the planet's physical properties, the body's orientation with respect to the planet, and the way the body's failure is characterized. Because of these last two dependencies, there are several estimates of the Roche limit.

Davidsson (2001) evaluated the Roche limit as a function of the encounter velocity and periapse distance, when the body's long-axis points towards the planet at periapse, i.e., $\phi_{q}=0^{\circ}$. This estimate is shown in Fig. 5. Davidsson (2001) required the failure to occur along pre-defined planes of the body. This contrasts with the way that we model the body's initial failure, where the body breaks apart in a "volume-averaged" manner, i.e., we do not presuppose a particular plane of failure. However, it is possible to apply the volume-averaged methodology to a rigid-granular ellipsoid and obtain appropriate Roche limits. Fig. 5 shows that the Roche limit obtained by the volume-averaged approach for $\phi_{q}=0^{\circ}$ is the same as Davidsson's (2001). This gives us confidence to employ the volume-averaged approach to obtain Roche limits for other longaxis orientations, and the results for several other $\phi_{q}$ are indicated in Fig. 5. This is necessary because in our case failure may be initiated at non-zero values of the long-axis orientation $\phi_{q}$, and such cases are not considered by Davidsson (2001). From Fig. 5, we see that the Roche limit curves vary significantly depending on the asteroid's orientation. The details for calculating this more generalized Roche limit appropriate for solid objects and derived using the volume-averaged approach are not pursued here, and will be published separately.

In general, for a fixed shape, if a body's planetary distance and spin are such that it lies outside the curve corresponding to $\phi_{q}=90^{\circ}$ it will definitely fail; if it lies inside the curve corresponding to $\phi_{q}=0^{\circ}$, it will never fail; and finally, if it lies between the two curves, its failure depends on its orientation at closest approach.

We should stress that the Roche limit discussed here does not depend on the asteroid's encounter velocity as it is calculated for static conditions, neglecting the asteroid's changing parameters during the fly-by. In particular, while the limit includes the effects of stresses due to tides, spin and internal gravity, it does not follow changes in "centrifugal" stresses due to the action of tidal torques on the asteroid's spin. Scheeres et al. (2000) and Scheeres (2001) investigate the effects of the tidal torques on the asteroid's spin in detail, and a truly complete Roche limit analysis would incorporate their results, thereby accounting for the change in the Roche limit as the asteroid's spin rate changes during its fly-by. However, such an analysis is not yet available and we will attempt to interpret our results in terms of the static landscape defined by the curves of Fig. 5.

Fig. 6 provides another way to look at the Roche limit. It shows the Roche 


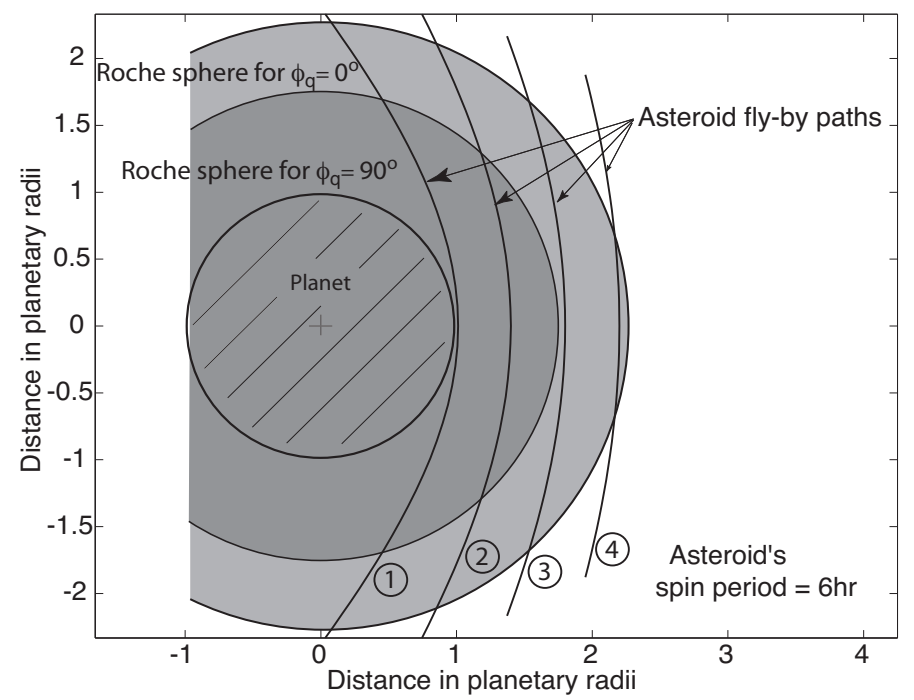

Fig. 6. A schematic of possible asteroid paths through the inner and outer Roche spheres. All the fly-bys shown had $v_{\infty}=3.26 \mathrm{~km} / \mathrm{s}$, while the object's spin period was fixed at six hours

spheres ${ }^{1}$ corresponding to $\phi_{q}=90^{\circ}$ and $\phi_{q}=0^{\circ}$ for the same asteroid as in Fig. 5. Several potential fly-by paths are also drawn. We call the part of the asteroid's fly-by path that intersects any of the Roche spheres, the "failure zone". Thus, if the failure zone of a fly-by path intersects both the inner and the outer Roche spheres, as for paths 1 and 2, all asteroids on those fly-by paths will fail with a high probability. On the other hand, paths 3 and 4 do not intersect the inner Roche sphere. This means that it is possible for the asteroid to traverse the failure zone without ever failing. Further, Fig. 6 hints at other possibilities. For example, the failure zone of path 4 is rather small, with the object spending only about 30 minutes in this zone, much shorter than its spin period of six hours. Thus, if the tidal torque due to the planet manages to decrease the asteroid's spin during its passage through periapse, it will shrink the size of the Roche spheres, implying that the asteroid will slip past without failing. Similarly, an increase in the spin of an asteroid on path 3 may cause it to fail at orientations that are precluded by an initial spin period of six hours. We explore all these aspects in more detail below. Finally, as we mentioned above, the disruption subsequent to failure depends significantly on the quadrupole moment $\boldsymbol{M}_{\boldsymbol{Q}}$. It is difficult to follow the quadrupole moment once the body begins to deform. However, the extent of the failure zone (see Fig. 6) provides an estimate of the importance of the quadrupole moment during any particular fly-by.

$\overline{1}$ Spherical regions around the central planet indicated by the Roche limit, objects within which will fail due to tides. 


\subsection{Further analysis of fly-bys}

In Fig. 4 the overall gross trend, as $v_{\infty}$ and/or $q$ increases, is for the intensity of disruptions to lessen, until there are no break-ups. Consider first the effect of raising the periapse distance at a fixed encounter velocity $v_{\infty}$. Fig. 6 shows that as the asteroid moves further out, the failure zone's extent lessens. Thus, though failure is initiated for all encounters with $q \leqslant 2 R_{\oplus}$, the likelihood of subsequent disruption drops. Recall that the classical Roche limit does not depend on the encounter velocity.

Now consider keeping the periapse distance $q$ fixed and raising the encounter velocity $v_{\infty}$. In general, all asteroids still undergo initial failure for $q \leqslant 2 R_{\oplus}$. However, the extent of subsequent deformation depends on the encounter velocity. This is because at higher encounter velocities the asteroid does not spend enough time in the disruption zone, i.e., its interactions with the planet are limited. This is marginally accentuated by the fact that with higher encounter velocities, fly-by paths are straighter, shortening the failure zone. Thus, the severity of the disruption decreases as the encounter velocity increases at a fixed $q$. For each case, failure is not initiated in general when $q \geqslant 2 R_{\oplus}$, so that the asteroid passes by unaffected.

We now explore the effects of the long-axis' orientation at periapse $\left(\phi_{q}\right)$, which is responsible for different outcomes despite the same encounter velocity $v_{\infty}$ and periapse distance $q$, as seen in Fig. 4. The orientation $\phi_{q}$ affects both the magnitude of the tidal stresses and the tidal torques.

- Effect on tidal torques: Spin up/down: Because the asteroid is asymmetric about the 3 -axis, the planet can exert tidal torques. They can be obtained from the axial vector corresponding to the anti-symmetric part of $M_{Q}$ (see Sec. 5.2). These torques can raise or lower the asteroid's spin during its flyby, depending on the long-axis' orientation in the vicinity of the periapse. Expressions that estimate the change in rotation rate for rigid asteroids may be obtained from Scheeres (2001).

A larger spin translates into an augmented deformation for already disrupting asteroids, as the disruptive centrifugal "force" is increased. Tidal torques will be most effective when the long-axis points ahead or behind the planet as suggested by the two sketches within Figs. 8 and 11(a). For example, the amount spin-up (-down) of a prograde rotator due to a tidal torque that, say, is felt only at periapse, is maximized at $\phi_{q}=-45^{\circ}\left(45^{\circ}\right)$. Thus, if spin changes due to tidal torques were the only, or at least the dominant mechanism determining the disruption process, we would predict the deformation to be maximum when the long-axis' orientation at periapse $\phi_{q} \sim-45^{\circ}$, and least when $\phi_{q} \sim 45^{\circ}$.

Finally, we recall that tidal torques can be zero while the quadrupole 
moment $\boldsymbol{M}_{\boldsymbol{Q}}$ itself is non-zero. This happens when the object is oblate, or when $\phi_{q}=0^{\circ}$ or $90^{\circ} . \boldsymbol{M}_{\boldsymbol{Q}}$ captures also the tide-raising part associated with the difference between gravitational and centrifugal forces for an object with spatial extent.

- Effect on tidal stresses: As we note from Fig. 5, the long-axis' orientation at periapse affects the Roche limit; the critical periapse distance, i.e., the distance above which failure is not initiated, decreases with an increasing $\phi_{q}$ for a given spin. In other words, if we neglect changes in its spin, or, if tidal stresses were the principal mechanism for disruptions during its fly-by, depending on its long-axis' orientation at periapse, an asteroid with the same $v_{\infty}$ and $q$, may not fail $(\mathrm{N})$, fail with mild deformation $(\mathrm{M})$, or fail with significant deformation (S). Thus, for example, a tidal-stress analysis would predict that the asteroid with a six-hour period and at $q=1.8 R_{\oplus}$ in Fig. 5 will fail if, during its fly-by, its long-axis points towards the planet, but not if its long-axis is perpendicular to the position vector of the planet. Also, there will be an orientation, e.g., $\phi_{q}=60^{\circ}$, so that the corresponding Roche limit curve passes close to the test object, and the disruption subsequent to failure is mild.

These mechanisms above are, in some sense, antagonistic. This is because, while the disruptive effects of tidal stresses are more dramatic if at periapse the long-axis points towards the planet, i.e., $\phi_{q}=0^{\circ}$, tidal-torque-induced spin changes are extremized when $\phi_{q} \sim \pm 45^{\circ}$. This complicated interplay between tidal stresses and torques makes an analysis based purely on the Roche limit, as derived here, rather intricate. However, a complete Roche limit analysis that has woven into it the effects of changing spins and tides, and that directly indicates whether a fly-by is spin- or tidal-stress-controlled is unavailable. Thus, instead, we will explore the interplay between these two mechanisms via three test cases below. We will find that $\phi_{q}$ 's effect on both tidal stresses and tidal torques is important.

- Spin-controlled regime: Fig. 7 plots the final axes ratios versus the long-axis' orientation at periapse $\phi_{q}$ of fly-by tests carried out for a fixed $q$ of $1.8 R_{\oplus}$ and $v_{\infty}$ of $3.26 \mathrm{~km} / \mathrm{s}$, differing only in the long-axis' initial orientation. The asteroid's position with respect to Roche limits is indicated by test case A in Fig. 5.

From the plot we note that the severity of disruption, as indicated by lowered values of the final axes ratios $\alpha$ and $\beta$, increases as the asteroid's long-axis gets aligned towards the planet, i.e., as $\phi_{q} \rightarrow 0^{\circ}$, and continues to grow until $\phi_{q} \sim-45^{\circ}$. This is in keeping with the hypothesis that the asteroid's spin increased most when $\phi_{q} \sim-45^{\circ}$, and this boost in spin accentuates the subsequent disruption. Similarly, when $\phi_{q}$ is positive, the asteroid's rotation is slowed, causing a reduction in the observed deformation. This is confirmed by Fig. 8 in which we compare the spin histories 


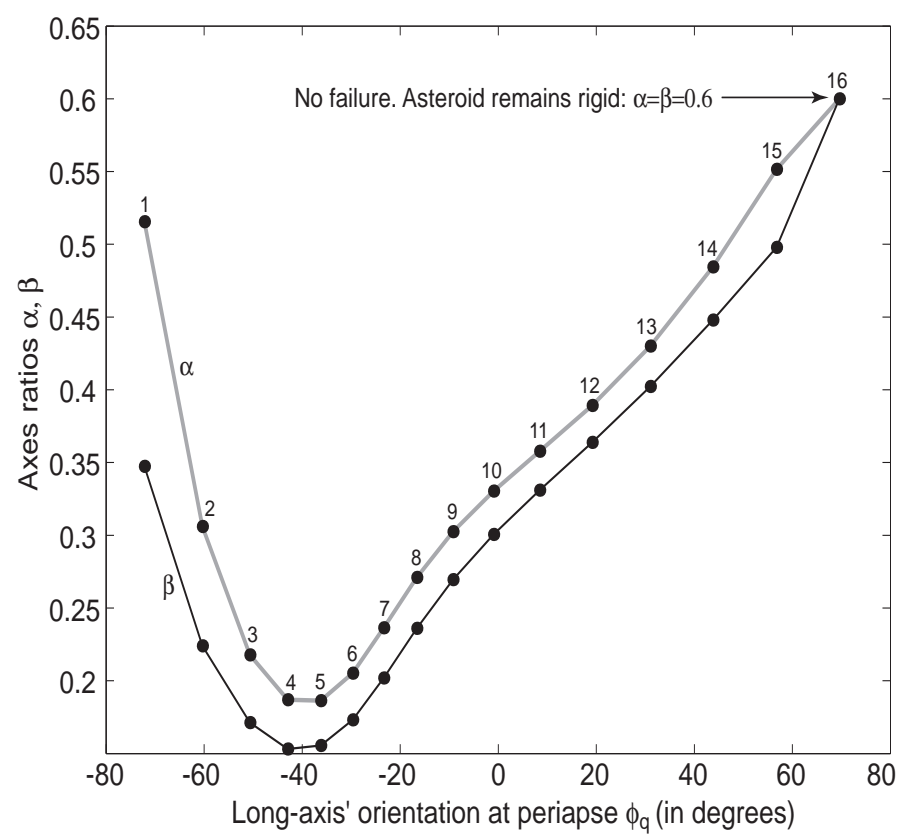

Fig. 7. Final axes ratios $\alpha=a_{2} / a_{1}$ (in gray) and $\beta=a_{3} / a_{1}$ (in black) are plotted against the corresponding long-axis' orientation at periapse $\phi_{q}$. The dots indicate individual tests that are identified by numbers next to them. Each fly-by had $v_{\infty}=3.26 \mathrm{~km} / \mathrm{s}$ and $q=1.8 R_{\oplus}$. The asteroid's initial spin period was six hours and it was in prograde motion. This figure should be compared to Fig. 9.

of test cases 10, 14 and 4 where $\phi_{q}$ is around $0^{\circ}, 45^{\circ}$ and $-45^{\circ}$ (obtained from Fig. 7). As Fig. 8 shows, the asteroid's spin is initially reduced as the body approaches periapse in cases 10 and 14 due to its orientation and that causes detrimental tidal torques. Meanwhile, due to the asteroid's favorable orientation in case 4 , the tidal torques help to augment the spin initially, which in turn induces larger deformations.

In contrast, a tidal-stress argument would predict maximum deformation when $\phi_{q} \sim 0^{\circ}$, and similar behavior for positive and negative values of $\phi_{q}$, because the Roche limit does not depend on $\phi_{q}$ 's sign. It turns out that, at the reasonably fast spin corresponding to a period of six hours, the "centrifugal" stresses play a dominant role, so that changes in spin due to tidal torques are more important than modifications in tidal stresses due to the alignment of the long-axis. In this sense, we term this as a spin-controlled regime.

- Tidal-stress-controlled regime: In Fig. 9 we plot the final axes ratios against the corresponding long-axis' orientation at periapse $\phi_{q}$ for fly-by tests carried out with the same $q$ and $v_{\infty}$ as in the case above, but with the asteroid's initial spin period lengthened (test case B in Fig. 5). We see that now the maximum deformation occurs at $\phi_{q} \sim 0^{\circ}$, with the deformation reducing with increasing $\left|\phi_{q}\right|$. This is explained by the fact that tidal stresses surge with decreasing $\left|\phi_{q}\right|$. In this case the spin is low enough for "centrifugal" stresses to be unimportant compared to tidal stresses. Thus, we call this 


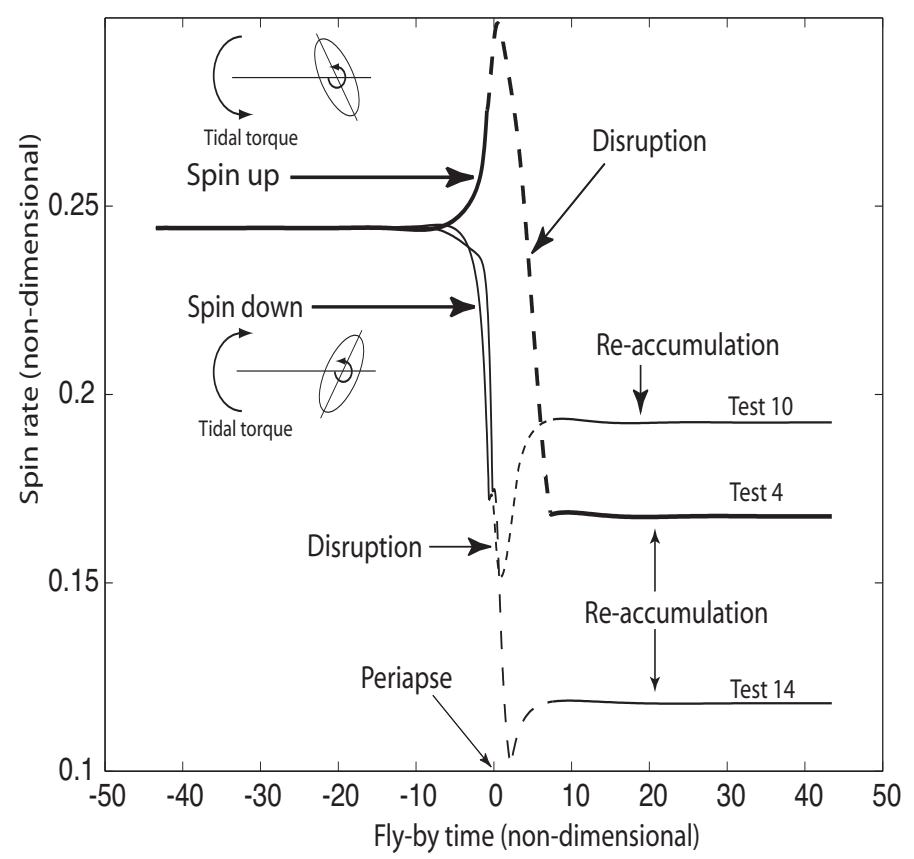

Fig. 8. The spin rate's evolution for fly-bys that have the same $q\left(=1.8 R_{\oplus}\right)$ and $v_{\infty}(=3.26 \mathrm{~km} / \mathrm{s})$, as in Fig. 7 . The initial spin period of the asteroid was six hours (or 0.24 in non-dimensional spin-rate units).

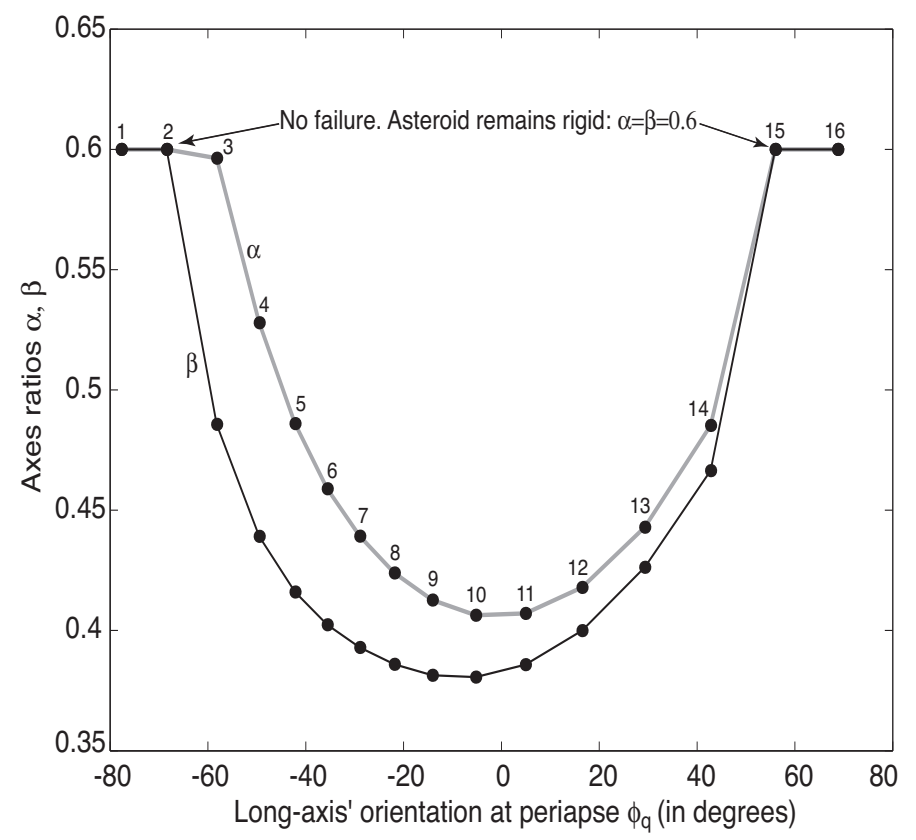

Fig. 9. Final axes ratios $\alpha=a_{2} / a_{1}$ (in gray) and $\beta=a_{3} / a_{1}$ (in black) are plotted versus the corresponding long-axis' orientation at periapse $\phi_{q}$. The dots indicate individual tests that are identified by numbers next to them. Each fly-by was characterized by $v_{\infty}=3.26 \mathrm{~km} / \mathrm{s}$ and $q=1.8 R_{\oplus}$. The asteroid's initial spin period was 12 hours and it was in prograde motion; cf. Fig. 7. 


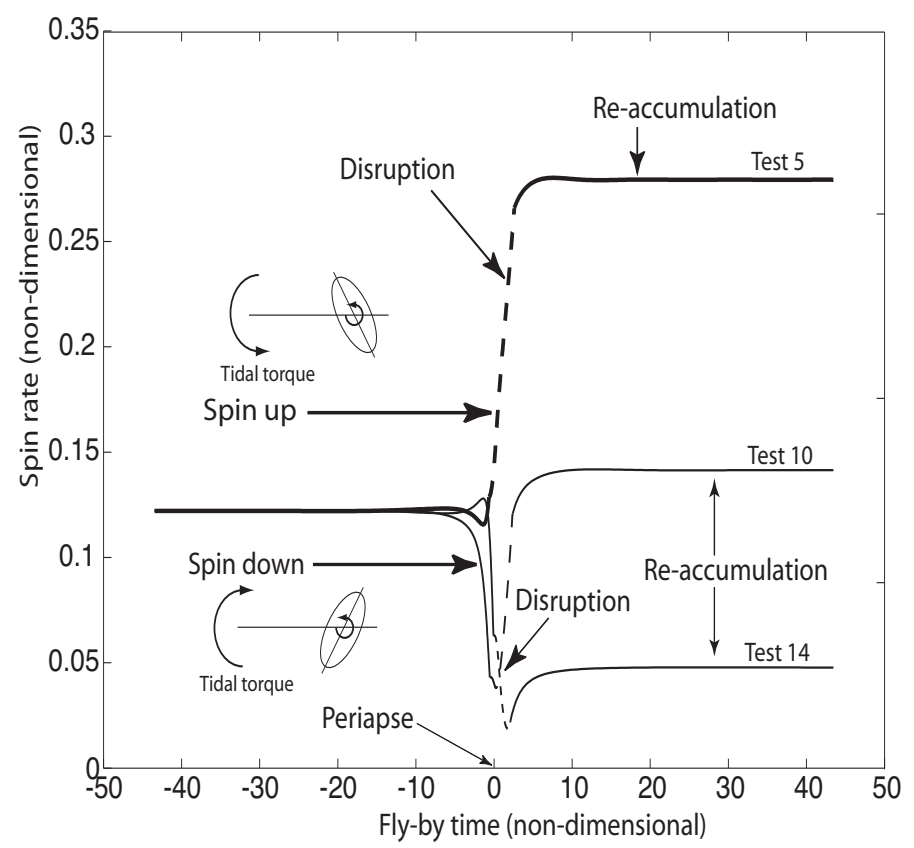

Fig. 10. The spin-rate's evolution for fly-bys that have the same $q\left(=1.8 R_{\oplus}\right)$ and $v_{\infty}(=3.26 \mathrm{~km} / \mathrm{s})$, as in Fig. 9. The initial period of the asteroid was 12 hours (or 0.12 in non-dimensional spin-rate units).

a tidal-stress-controlled regime. The fact that we do not quite recover a symmetric deformational behavior, as would be the case for a pure tidalstress-controlled fly-by, is because of the action of tidal torques. Indeed, spin-up induces greater deformation for $\phi_{q}<0^{\circ}$, and conversely, an adverse tidal torque retards deformation for $\phi_{q}>0^{\circ}$. Fig. 10 plots the spin histories of test cases 5, 10 and 14 from Fig. 9. This figure confirms the fact that even though the rotation rate of the asteroid increases in test case 5 $\left(\phi_{q} \sim-45^{\circ}\right)$, and decreases initially in test case $10\left(\phi_{q} \sim 0^{\circ}\right)$, Fig. 9 reports greatest deformation for test case 10 . Thus, it is the tidal stresses that play a greater role in determining the outcome of this class of fly-bys.

- Spin-initiated failure: A growth in an asteroid's spin can also initiate failure in asteroids that would otherwise have flown by as rigid objects. This can happen if the tidal torques can augment the asteroid's spin enough to push it outside its failure curve in Fig. 5. This is demonstrated in Fig. 11, where we consider four separate instances of an asteroid's fly-by that vary only in the long-axis' initial direction, leading in turn to different orientations during passage through periapse. This particular fly-by is indicated by test case $\mathrm{C}$ in Fig. 5.

First, consider the fly-bys numbered 2, 3 and 4. Fig. 11(a) shows that asteroids in these fly-bys are so aligned near periapse that tidal torques tend to increase their spin. In case 2 the spin is raised, but not enough to initiate failure, as seen by the corresponding curve for the internal stress in Fig. 11(b), where the stresses have been non-dimensionalized by $(3 / 20 \pi)\left(2 \pi \rho_{s} G m_{A}\right)$ $\left(4 \pi \rho_{A} / 3 m_{A}\right)^{1 / 3}$. Thus, the asteroid passes by as a rigid object, though its 
spin has grown substantially as a result of the encounter. In cases 3 and 4, on the other hand, the initial augmentation in spin is enough to initiate failure. This is confirmed by the stresses becoming tensile in Fig. 11(b). Post-failure, the asteroid is governed by a granular rheology, and is allowed to deform, as is indicated by the dashed curves 3 and 4 . Once the asteroid leaves the vicinity of periapse, it begins to re-accumulate until it becomes rigid again, when the volume fraction reaches a critical value; thus in Fig. 11, curves 3 and 4 become solid again. It is interesting to see from Fig. 11(a) that the asteroid's spin drops from the value it had attained just before failure. This happens because the deformation augments the inertia about the spin axis, mandating a slower spin in order to balance angular momentum. Finally, we caution that when the asteroid is no longer rigid, its spin is no longer given by the axial vector associated with the tensor $\boldsymbol{W}$. Thus, the dashed part of the curve, which follows the evolution of $\boldsymbol{W}$ 's axial vector, does not indicate the actual spin, which instead is defined by the rate at which the principal axes of the ellipsoid rotate.

A reduction in the spin's magnitude, on the other hand, has a stabilizing effect that reduces the amount of break-up, and may even suppress initial failure. That spin-down is indeed possible and leads to a lessening of the maximum principal stress is demonstrated by history 1 in Fig. 11.

The outcomes reported in Fig. 4 agree reasonably well with those obtained by Richardson et al. (1998). However, in contrast to the latter's results, our model does not predict disruption at higher values of the encounter velocity $v_{\infty}$ and periapse distance $q$. Moreover, we find less post-encounter deformation than that published by Richardson et al. (1998). For example, Richardson et al. (1998) predict $\mathrm{S}$ type encounters for $v_{\infty}$ 's greater than $15 \mathrm{~km} / \mathrm{s}$ at $q=1.01 R_{\oplus}$, while we, at best, predict only M-type encounters. The asteroid spends about 12 minutes inside the failure zone in these encounters. Recall that our classification scheme does not differentiate between the S- and B-type encounters of Richardson et al. (1998); we label them both as S-type encounters. Similarly, at $q$ 's of $2.6 R_{\oplus}$ and $3 R_{\oplus}$, where the maximum tidal stresses are of the order of $0.23 \mathrm{kPa}$ and $0.35 \mathrm{kPa}$, respectively, we forecast that the fly-by has little or no effect on the asteroid, while Richardson et al. (1998) predict modification, at least at low encounter velocities. Note that the classical Roche limit, calculated assuming our asteroid to be fluid, is about $3.4 R_{\oplus}$. This underlines the fact that our model, and that of Richardson et al. (1998), are resistant to shear.

These differences occur for two important reasons:

- Failure criterion: Richardson et al. (1998) represent the asteroid as an aggregate of smooth spheres. In order to model such an aggregate as a rigidgranular material with a failure law, one must pick an appropriate failure criterion. In the above, we chose a tensile failure criterion, where the ma- 


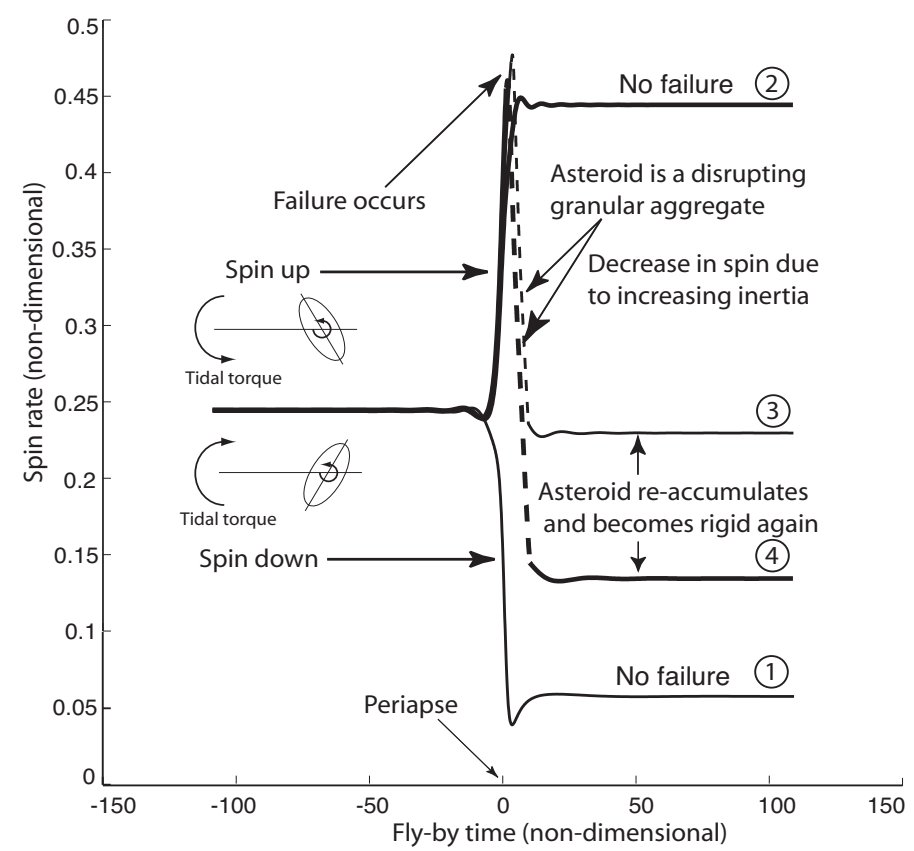

(a) The evolution of the spin.

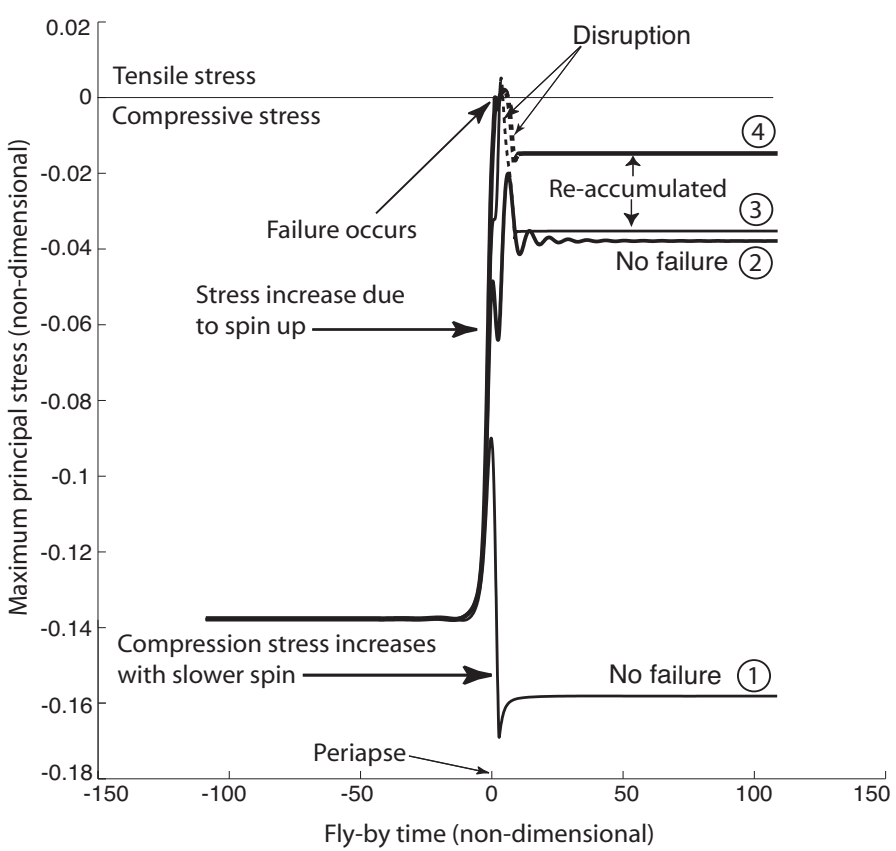

(b) The evolution of the maximum principal stress.

Fig. 11. Evolutions of the spin rate and maximum stress for four different long-axis orientations, but the same fly-by conditions $\left(v_{\infty}=1.1 \mathrm{~km} / \mathrm{s}\right.$ and $\left.q=2.3 R_{\oplus}\right)$. The initial rotation was prograde with a period of six hours. Effects of the Earth's presence are felt over a period of approximately seven hours, i.e., about 30 units of non-dimensional time, during which the asteroid is within a distance of about 20 planetary radii. Compared to Figs. 8 and 10 a larger window of time is shown. 
terial failed if any principal stress became tensile. However, even when all principal stresses are compressive, the aggregate can fail if a shear stress, on some plane in its interior, manages to overcome the resistance due to the interlocking of the spheres. It is possible to model this interlocking as an internal geometric friction, i.e., a frictional resistance whose origins lie in the aggregate's arrangement, rather than in the surface properties of its constituent spheres. Thus, an appropriate failure criterion governing the transition from a rigid state, where the spheres are locked together, to a more mobile granular state, could be the Mohr-Coulomb failure criterion (Sec. 4.2) with some friction angle $\phi_{F}$. Holsapple (2001), Richardson et al. (2005) and Sharma et al. (2005) provide further endorsement for using this method to describe the aggregate considered by Richardson et al. (1998).

Holsapple (2001) studied the equilibrium shapes of spinning ellipsoids that were rigid until they failed according to the Mohr-Coulomb failure condition. Using an exact plasticity analysis, he was able to map out regions that depended on the ellipsoid's spin and its shape, as defined by the axes ratios $\alpha$ and $\beta$, within which spinning ellipsoids could exist without failure. Subsequently, Richardson et al. (2005), when considering equilibrium shapes of spinning, ellipsoidal, granular aggregates similar to the ones used by Richardson et al. (1998), showed that the ellipsoidal aggregates that remained in equilibrium all clustered together. According to Holsapple's (2001) study, this region was described by a rigid-plastic ellipsoid with a Mohr-Coulomb failure criterion and an internal friction angle $\phi_{F}$ of $40^{\circ}$.

Using a Mohr-Coulomb criterion, which, as mentioned previously, permits earlier failure than the tensile criterion, should have the effect of increasing the frequency and severity of disruptions by making the asteroid more susceptible to break-ups. Thus, the asteroid will pass from a rigid state into a granular state, where it can be deformed, much more easily. Below we see that this hypothesis is indeed partially true.

Another reason that we obtain fewer break-ups than Richardson et al. (1998) is the way that we model the reverse transition of the asteroid from a granular to a rigid state. As mentioned earlier, we assume that the asteroid's state is rigid once the volume fraction in the granular state crosses a cut-off limit, which is typically set at around the volume fraction of a random, closely packed aggregate of identical spheres. If we imagine that the asteroid is made up of rock chunks held together by self-gravity alone, then it is plausible that over time, due to gravitational pressure and adhesive interactions, these rocks become sintered together. Thus, the asteroid appears, for low-enough stresses, to behave rigidly. This, in a way, was one of the motivations for choosing an initially rigid state in the problem above.

Now suppose that such an asteroid undergoes an event, e.g., a planetary fly-by, which breaks apart any bonds that might have formed between its constitutive rock chunks. Then it is unlikely that these internal bonds, which probably developed over long periods of sustained pressure, will be immediately regenerated if the asteroid becomes dense enough during the fly-by. 
Thus, by assuming that the asteroid becomes rigid if the volume-fraction of the granular state increases sufficiently, we probably lessen the number of break-up events.

The above deficiency can be overcome by not allowing the asteroid to become rigid again once it has passed into a granular state. But so far an efficient continuum model for the dynamics of a granular material at high volume fractions is not available, and this remains a problem for future study.

- Volume-averaging: In the volume-averaged models used in the above analysis, an object will fragment only if its average stress satisfies some failure criterion. Thus, our approach will miss cases in which the body fails at certain points but not on average. Richardson et al. (1998) use a numerical scheme to follow all the constituent spheres in their model, and so, in fact, are ascertaining the body's failure point-wise. They find, for example, that a gravitationally held aggregate will frequently start shedding mass from its surface before the failure reaches the interior. This was also seen by Asphaug et al. (1994) and seems intuitively plausible. Such surface-shedding cannot, of course, be captured by our volume-averaged model. We could improve our model by perhaps applying volume-averaging over layers rather than the full volume, but this would require a modification of the governing equations with surface terms coming into play at the boundaries between the layers. However, as we remark later, other reasons may account for most discrepancies between our results and previous simulations.

The possibility of localized failure is increased at encounter velocities significantly higher than the speed of sound in the asteroid's material. This is a result of the stress information not being communicated to the asteroid's interior fast enough to dissipate high local stresses, leading to failure. This may not be captured by the volume-averaged approach because the stress on average may be lower than that required for failure.

\subsection{Outcomes with the Mohr-Coulomb criterion}

When the Mohr-Coulomb criterion (30) with a friction angle $\phi_{F}$ of $40^{\circ}$ is used to govern the transition from a rigid to a granular state, previously unaffected asteroids begin to break apart. Furthermore, the fraction of asteroids that disrupt for particular choices of $q$ and $v_{\infty}$ also increases, reflecting the relative ease with which asteroids can now fail. However, in the case of an asteroid that is already deforming when the tensile criterion was used, there is little enhancement in its distortion. This occurs because the extent of deformation depends on the time the asteroid spends disrupting, i.e., the interval between failure's initiation and the asteroid's re-accumulation. Whenever passage through periapse happens rapidly, this interval differs little between the two failure criteria, even though failure occurs earlier when the Mohr-Coulomb 


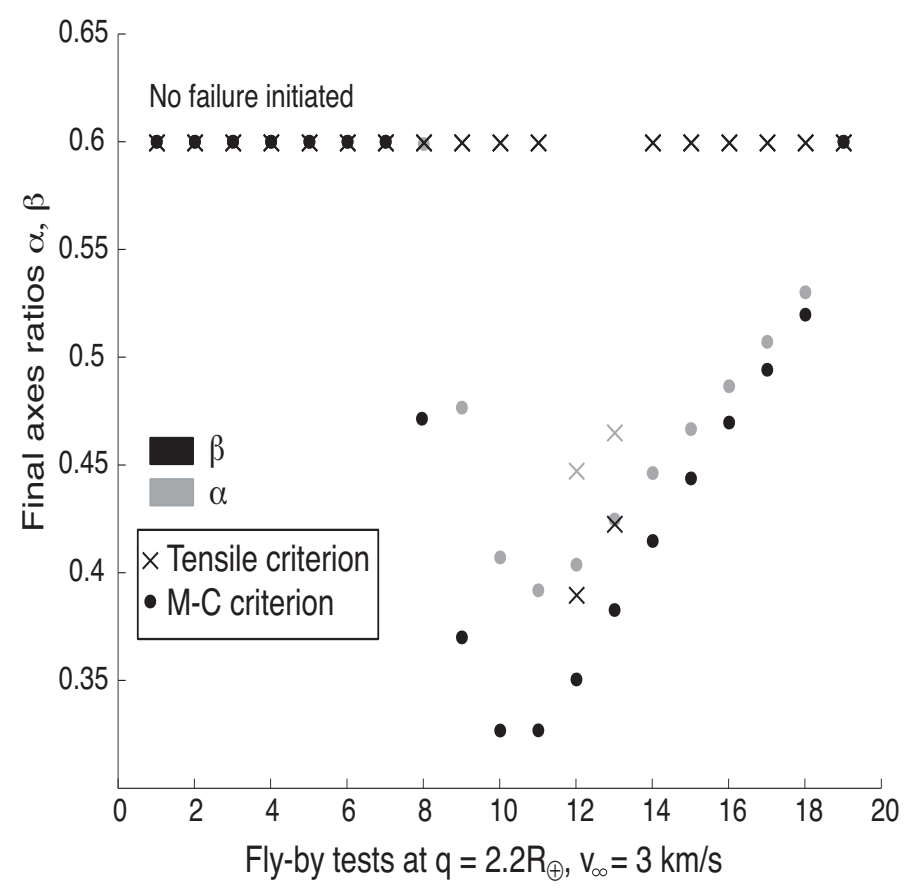

Fig. 12. A comparison of final axes ratios for fly-bys using the tensile and the Mohr-Coulomb (M-C) failure criteria.

criterion is employed.

Fig. 12 tabulates the final shapes of 18 fly-bys that differ only in the long-axis' orientation at periapse $\phi_{q}$; they all have the same periapse distance $q=2.2 R_{\oplus}$ and encounter velocity $v_{\infty}=3 \mathrm{~km} / \mathrm{s}$. Note that in contrast to previous figures, in Fig. 12 we plot the axes ratios against individual tests and not $\phi_{q}$. When the tensile failure criterion was employed, the asteroid's final shape was unchanged in $90 \%$ of the cases. However, once we utilize the Mohr-Coulomb criterion, the number of disruptions increases significantly as does the deformation. From Fig. 12 we observe that the asteroid failed in only two cases when the tensile criterion was employed. Note though that in these cases too the final axes ratios were greater than the corresponding ones obtained when the MohrCoulomb criterion was utilized, thus indicating lesser deformation during the fly-by. Finally, we mention that as we lower the internal friction angle $\phi_{F}$, the number and intensity of disruptions grow apace. We point out that in these computations, the numerical approach and the initial conditions were exactly the same as before.

\subsection{The effect of spin direction}

We also investigated fly-bys where the asteroid had an initial retrograde spin. For outcomes of retrograde fly-bys in the spin-controlled regime, where the 


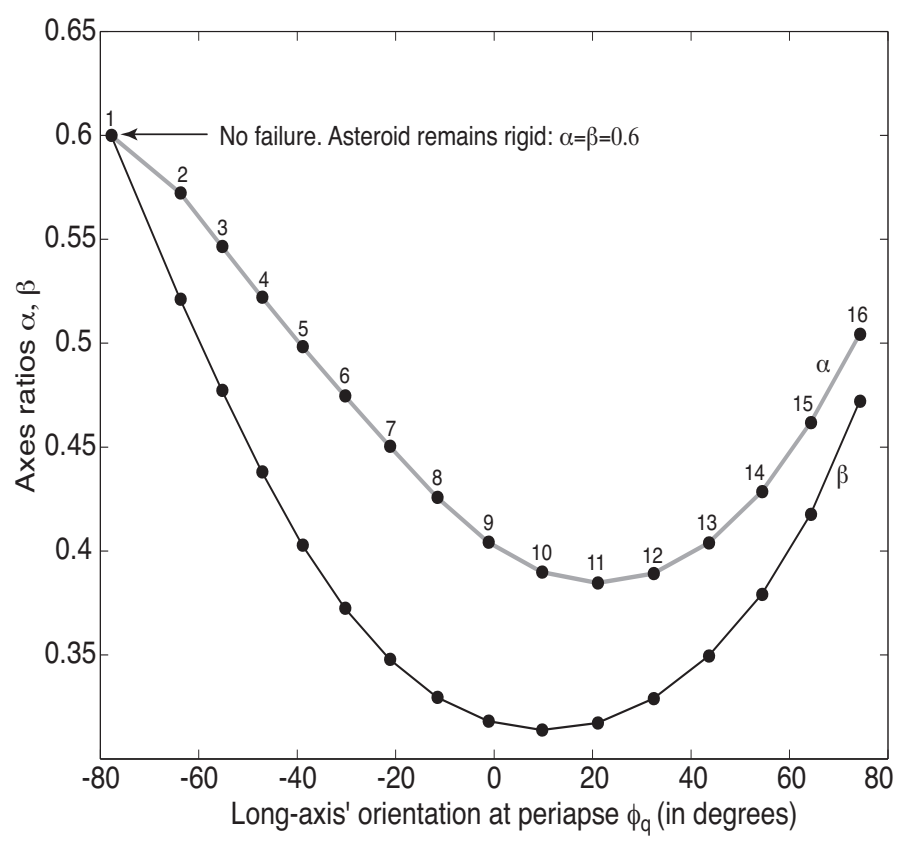

(a) Initial spin period of six hours: Spin-controlled regime.

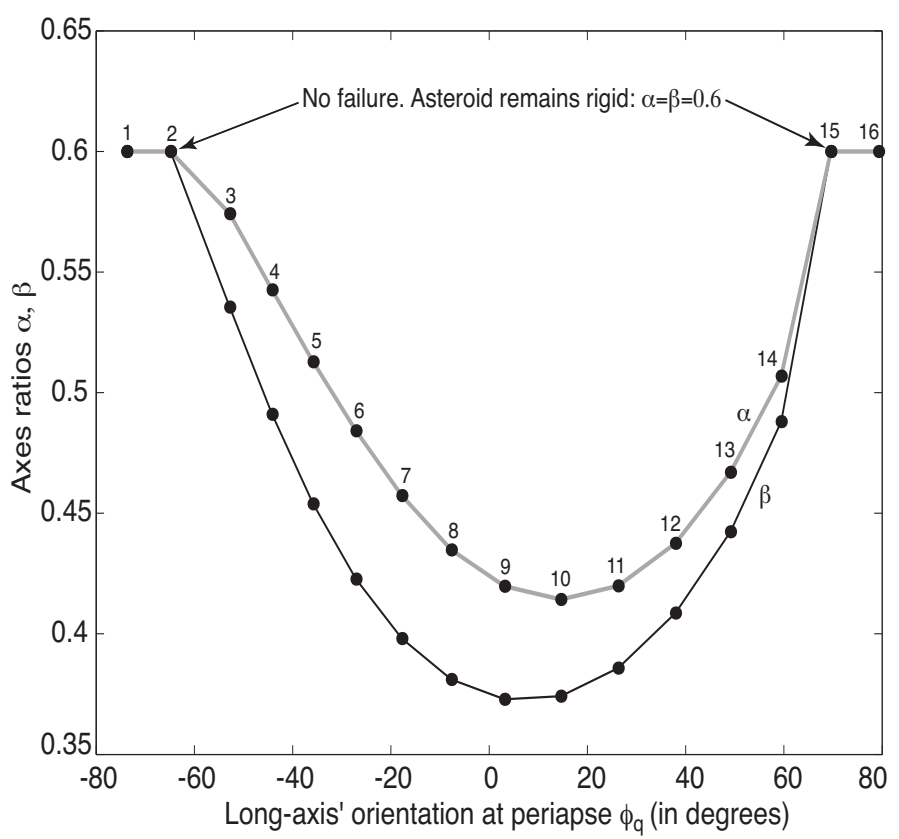

(b) Initial spin period of twelve hours: Tidal-stress controlled regime.

Fig. 13. Final axes ratios $\alpha=a_{2} / a_{1}$ (in gray) and $\beta=a_{3} / a_{1}$ (in black) for retrograde fly-bys are plotted against the corresponding long-axis' orientation at periapse $\phi_{q}$. The dots indicate individual tests that are identified by numbers next to them. The fly-bys had $v_{\infty}=3.26 \mathrm{~km} / \mathrm{s}$ and $q=1.8 R_{\oplus}$. 
asteroid's spin plays an important role, Fig. 13(a) shows a decrease in the number and the severity of disruptions. This agrees with the observation of Richardson et al. (1998). Indeed, comparing Fig. 13(a) with the outcomes of corresponding prograde fly-bys shown in Fig. 7, we see that disruption, as characterized by the change in $\alpha$ and $\beta$, is much less in the retrograde case. It is interesting to note however that the shapes of the $\alpha$ and $\beta$ curves exhibit a degree of mirror symmetry. This may reflect the fact that, in a spin-controlled regime, the deformation in a prograde fly-by is maximized when $\phi_{q} \sim-45^{\circ}$, but in the retrograde case it should occur when $\phi_{q} \sim 45^{\circ}$. As explained below this is not strictly true owing to the mechanics of a retrograde fly-by being fundamentally different from a prograde one.

A similar reduction in the disruption was not observed for retrograde fly-bys that were tidally controlled, i.e., where the spin was low enough for tidal stresses to dominate. Indeed, the outcomes of such retrograde fly-bys shown in Fig. 13(b) are almost the same as the results of analogous prograde flybys (Fig. 9). This behavior may be easily explained by utilizing a Rochelimit analysis at the periapse. To see this, we simply note that the centrifugal "force", the only term that varies with the spin, depends only on the spin's magnitude, and not its sign. Thus, there is no difference between Roche limits for prograde and retrograde fly-bys, leading to similar behavior. Note that because the fly-by is tidally controlled, we can neglect the effect of changes in spin rate.

An explanation of the fewer number of disruptions for prograde fly-bys, in situations where the asteroid's spin plays a crucial role, requires more understanding. In this case, a Roche-limit analysis carried out at periapse is found to be insufficient as it predicts similar behaviors for prograde and retrograde fly-bys. In the spin-controlled regime, changes in spin play an important role, and these changes cannot be accounted for by considering only the encounter geometry at periapse. Indeed, such an analysis would predict that an asteroid rotating in a prograde fashion will be spun up (down) at a $\phi_{q}$ of about $-45^{\circ}\left(45^{\circ}\right)$, whereas the same asteroid at those $\phi_{q}$ 's would be spun down (up) if rotating retrograde. This would further imply that the statistics of disruptions would not change, because for every fly-by in which a prograde rotator is spun-up at $\phi_{q} \sim-45^{\circ}$, there will be a corresponding fly-by with $\phi_{q} \sim 45^{\circ}$, where a retrograde spinner gets speeded up. We would then expect the outcomes of retrograde fly-bys to look similar to Fig. 7 with the maximum deformation occurring at $\phi_{q} \sim 45^{\circ}$. This is not the case. Thus, in order to estimate an asteroid's changes in rotation we need to follow its history throughout the periapse's vicinity.

Such an analysis has been carried out by Scheeres (2001) in the context of rigid asteroids. He showed that for the same fly-by paths, a prograde rotator suffered much more drastic changes in spin than a retrograde one. Thus, his 


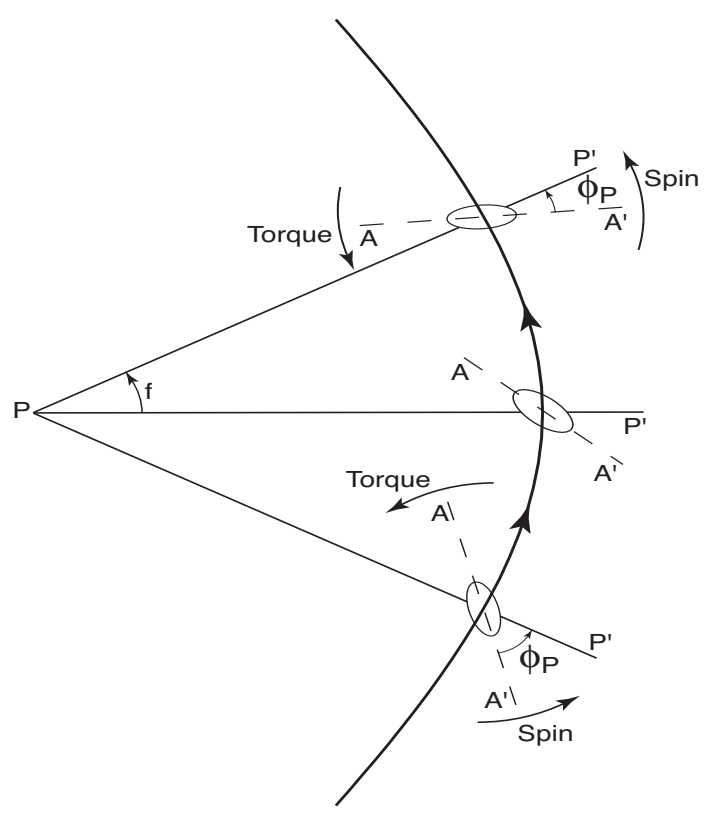

(a) Prograde fly-by

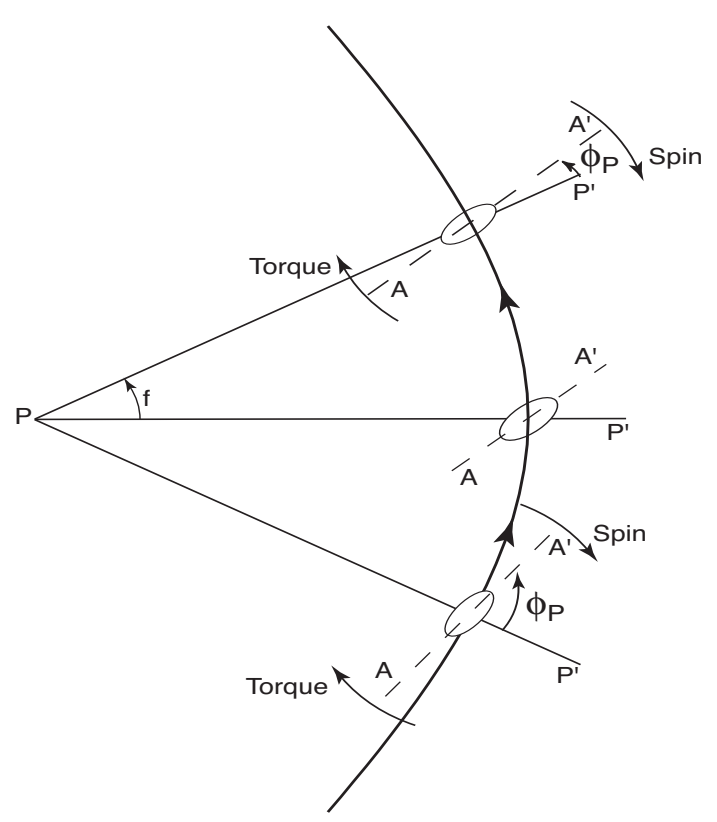

(b) Retrograde fly-by

Fig. 14. Schematics of two fly-bys differing only in the asteroids initial spin direction. Not shown is the angle $\theta$ that the asteroid's long-axis makes with the horizontal.

analysis predicts that prograde fly-bys will disrupt to a greater extent than retrograde ones. We will not repeat Scheeres' (2001) analysis here, but instead motivate the mechanics underlying his treatment through Fig. 14, which shows two configurations that guarantee spinning up of asteroids during prograde 
and retrograde fly-bys, respectively. Note our sign convention, wherein $\phi_{P}$ is measured from the line $A A^{\prime}$ to $P P^{\prime}$ in the prograde case, and from $P P^{\prime}$ to $A A^{\prime}$ for a retrograde fly-by. In either case, the angle $\phi_{P}$ yields the orientation of the asteroid's long-axis with respect to the central planet, and equals $\phi_{q}$ at periapse. From the sketch in Fig. 14 we see that at any point on its trajectory, the torque experienced by an ellipsoid (asteroid), taken to have its rotation normal to its orbit plane, is maximum for $\phi_{P}=45^{\circ}$. In order to change the asteroid's rotation the most from its planetary engagement, the largest angular impulse should be applied during its fly-by. This would be maximized if $\phi_{P}$ throughout the asteroid's passage were always $45^{\circ}$. However, Fig. 14 shows that

$$
\phi_{P}=f-\theta
$$

for prograde fly-bys, and

$$
\phi_{P}=\theta-f
$$

for retrograde ones, where $\theta$ is the angle made by the asteroid's long axis with the horizontal and $f$ is the true anomaly. Thus, $\phi_{P}$ generally varies throughout an encounter because both the orbital position changes and the asteroid rotates. Nonetheless, an asteroid's spin can still be augmented, e.g., we are assured of an increase in an asteroid's rotation if $\phi_{P}$ remains positive throughout the asteroid's planetary interaction so as to produce a positive torque.

If we now assume that the line $A A^{\prime}$ in Fig. 14 rotates at a rate $\dot{\theta}$, which is the asteroid's angular velocity, while the line $P P^{\prime}$ rotates at $\dot{f}$ then we can write $\phi_{P}$ 's rate of change for prograde fly-bys as

$$
\dot{\phi}_{P}=\dot{f}-\dot{\theta}
$$

while

$$
\dot{\phi}_{P}=\dot{\theta}-\dot{f}
$$

for retrograde fly-bys, which, because $\dot{\theta}<0$ for retrograde spins, becomes

$$
\dot{\phi}_{P}=-|\dot{\theta}|-\dot{f}
$$

From Eqs. (46) and (47) we see that in any particular encounter, because $\dot{f}$ is always positive, while $\dot{\theta}$ is positive for prograde but negative for retrograde, $\phi_{P}$ always changes more slowly for positive rotation than for retrograde. Thus, $\phi_{P}$ remains positive for longer periods during prograde fly-bys, augmenting the relative angle over which favorable tidal torques are "averaged" and thereby increasing the chances of an asteroid being spun up during such interactions. 
Hence, in any encounter a prograde-rotating body will be more affected than will a retrograde-spinning body.

Finally, it is interesting to note that if the asteroid's initial spin is very rapid, the integrated effect of the torques is small because the asteroid will experience both positive and negative torques during a single interaction. Thus, one would expect very little difference between retrograde and prograde encounters, and this has been pointed out recently by Scheeres et al. (2004). Furthermore, if at pericenter $\phi_{P}=45^{\circ}$, then the way to generate the greatest angular impulse is to keep $\phi_{P}$ as close to this same angle as possible, namely to have $\theta$ and $f$ rotate at roughly the same rates. Thus, the above analysis would predict that in order for angular impulse to be maximum in any encounter, it should not only act on a prograde-spinning body, but also $\dot{f}$ should roughly equal $\dot{\theta}$.

\subsection{Different initial spin magnitudes}

We have already explored how, depending on the spin, a fly-by may be tidalstress or spin-controlled. We further considered the effects of different initial spins for a fixed encounter velocity $\left(v_{\infty}=9 \mathrm{~km} / \mathrm{s}\right)$ and periapse distance $\left(q=1.8 R_{\oplus}\right)$. The results, as expected, show that there is an increase in disruptive encounters with faster asteroid spin rates. Thus, while a fly-by with a rotation period of three hours resulted in an S-type encounter, fly-by's with spin periods of six and nine hours resulted in $\mathrm{M} / \mathrm{N}$ - and N-type encounters, respectively. This is explained simply by the stronger centrifugal "force" tearing the asteroid apart for a faster spin; hence, the failure zone along the asteroid's path expands.

\section{Final remarks}

We have employed a very simple model using volume-averaged methods to investigate planetary fly-bys of asteroids. Our results agree well with previous discrete particle simulations (Richardson et al. 1998). We further saw how using a less stringent failure criterion would predict an increased number of disruptions.

Two reasons accounted for the few variations between previously simulated results and ours. First, the laws governing the transition of the asteroid between rigid and granular states were argued to be important. Additional considerations provided insight into the mechanics of break-ups. Moreover, the reverse transition (from a granular to a rigid state) is, perhaps, equally crucial and should be investigated further. 
Second, we discussed the approximations involved when using volume-averaged methods. This, however, may not be as important a factor as it seems at first glance. We have already noted how the volume-averaged method recovers verbatim Davidsson's (2001) results for the Roche limit. It is also possible to obtain Holsapple's (2001) results about equilibrium of spinning ellipsoids (Sharma et al. 2005). In particular, the assumption, inherent in a volumeaveraged approach, that the body fails simultaneously at all points was, in fact, seen to be the case by Holsapple (2001) and by Davidsson (2001). Thus, it seems that a volume-averaged theory may still provide good approximations in simple dynamical situations, such as planetary fly-bys. In summary, we have demonstrated that volume-averaged models are useful in studying the breakup and the rotational dynamics of deformable, ellipsoidal asteroids because these methods provide straightforward access to the underlying mechanisms responsible for disruption and/or deformation during planetary fly-bys.

\section{Acknowledgements}

We thank the reviewers Donald Korycansky and especially Daniel Scheeres whose comments improved our work. We would also like to thank Anthony Dobrovolskis for extremely helpful suggestions. JAB acknowledges the support of NASA-NNG5GG88G in the Planetary Geology and Geophysics program. JTJ acknowledges the support of NASA grant NCC3-797.

\section{References}

[1] E. Asphaug and W. Benz. Density of comet Shoemaker-Levy 9 deduced by modeling breakup of the parent "rubble pile". Nature, 370:120-124, 1994.

[2] E. Asphaug and W. Benz. Size, density and fracture of comet ShoemakerLevy 9 inferred from the physics of tidal breakup. Icarus, 121:225-248, 1996.

[3] S. Chandrasekhar. Ellipsoidal Figures of Equilibrium. Yale Univ. Press, New Haven, CT, 1969.

[4] S. Chapman and T.G. Cowling. The Mathematical Theory of Nonuniform Gases. Cambridge U. Press, Cambridge, 3rd edition, 1970.

[5] W.F. Chen and D.J. Han. Plasticity for Structural Engineers. SpringerVerlag, New York, 1988.

[6] B. Davidsson. Tidal splitting and rotational breakup of solid biaxial ellipsoids. Icarus, 149:375-383, 2001.

[7] Y.C. Fung. Foundations of Solid Mechanics. Prentice-Hall, 1965.

[8] K. Holsapple, I. Giblin, K. Housen, K. Nakamura, and E. Ryan. Asteroid 
impacts: Laboratory experiments and scaling laws. In W.F. Bottke Jr., A. Cellino, P. Paolicchi, and R.P. Binzel, editors, Asteroids III, pages 443-462. U. Arizona Press, 2002.

[9] K.A. Holsapple. Equilibrium configurations of solid cohesionless bodies. Icarus, 154:432-448, 2001.

[10] G.A. Holzapfel. Nonlinear Solid Mechanics. John Wiley and Sons, 2001.

[11] J. T. Jenkins and S. B. Savage. Theory for the rapid flow of identical, smooth, nearly elastic spherical particles. J. Fluid Mech., 130:187-202, 1983.

[12] J. T. Jenkins and C. Zhang. Kinetic theory for nearly elastic, slightly frictional spheres. Phys. Fluids, 14:1228-1235, 2002.

[13] O.D. Kellogg. Foundations of Potential Theory. Dover, New York, 1953.

[14] T.W. Lambe and R.V. Whitman. Soil Mechanics. John Wiley and Sons, New York, 1969.

[15] A.E.H. Love. A Treatise on the Mathematical Theory of Elasticity. Dover, New York, 4th edition, 1946.

[16] C.D. Murray and S.F. Dermott. Solar System Dynamics. Cambridge U. Press, Cambridge, 1999.

[17] D. C. Richardson, P. Elankumaran, and R. E. Sanderson. Numerical experiments with rubble piles: equilibrium shapes and spins. Icarus, 173:349-361, 2005.

[18] D.C. Richardson, W.F. Bottke Jr., and S.G. Love. Tidal distortion and disruption of earth-crossing asteroids. Icarus, 134:47-76, 1998.

[19] D.C. Richardson, Z.M. Leinhardt, H.J. Melosh, W.F. Bottke Jr., and E. Asphaug. Gravitational aggregates: Evidence and evolution. In W.F. Bottke Jr., A. Cellino, P. Paolicchi, and R.P. Binzel, editors, Asteroids III, pages 501-515. U. Arizona Press, 2002.

[20] D. J. Scheeres. Changes in rotational angular momentum due to gravitational interactions between two finite bodies. Celest. Mech. Dyn. Astr., 81:39-44, 2001.

[21] D. J. Scheeres, F. Mazari, and A. Rossi. Evolution of neo rotation rates due to close encounters with earth and venus. Icarus, 170:312-323, 2004.

[22] D. J. Scheeres, S. J. Ostro, R. A. Werner, E. Asphaug, and R. S. Hudson. Effect of gravitational interactions on asteroid spin states. Icarus, 147:106-118, 2000.

[23] I. Sharma, J. T. Jenkins, and J. A. Burns. Equilibrium shapes of ellipsoidal soil asteroids. In Proceedings of the 5th International Conference on Micromechanics of Granular Media, 2005.

[24] S. Sridhar and S. Tremaine. Tidal disruption of viscous bodies. Icarus, 95:86-99, 1992.

[25] S. Torquato. Nearest-neighbor statistics for packings of hard spheres and disks. Phys. Rev. E, 51:3170-3182, 1995.

[26] S. Torquato. Random Heterogeneous Materials. Springer-Verlag, New York, 1st edition, 2001.

[27] K. J. Walsh and D. C. Richardson. Binary near-earth asteroid formation: 
Rubble pile model of tidal disruptions. Icarus, 180:201-216, 2006. 